\title{
LNK/SH2B3 regulates IL-7 receptor signaling in normal and malignant B-progenitors
}

\author{
Ying Cheng, ${ }^{1,2}$ Kudakwashe Chikwava, ${ }^{3}$ Chao Wu, ${ }^{1}$ Haibing Zhang, ${ }^{1}$ Anchit Bhagat, ${ }^{1}$ Dehua Pei, ${ }^{4}$ John K. Choi, ${ }^{5}$ and Wei Tong ${ }^{1,2}$ \\ 'Division of Hematology, Children's Hospital of Philadelphia, Philadelphia, Pennsylvania, USA. ²Department of Pediatrics, University of Pennsylvania School of Medicine, Philadelphia, Pennsylvania, USA. \\ ${ }^{3}$ Department of Pathology, Children's Hospital of Philadelphia, Philadelphia, Pennsylvania, USA. ${ }^{4}$ Department of Chemistry and Biochemistry, The Ohio State University, Columbus, Ohio, USA. \\ ${ }^{5}$ Department of Pathology, St. Jude Children's Research Hospital, Memphis, Tennessee, USA.
}

\begin{abstract}
Philadelphia chromosome-like acute lymphoblastic leukemia (Ph-like ALL) is a high-risk ALL commonly associated with alterations that affect the tyrosine kinase pathway, tumor suppressors, and lymphoid transcription factors. Loss-offunction mutations in the gene-encoding adaptor protein LNK (also known as SH2B3) are found in Ph-like ALLs; however, it is not clear how LNK regulates normal B cell development or promotes leukemogenesis. Here, we have shown that combined loss of $L n k$ and tumor suppressors $T p 53$ or Ink4a/Arf in mice triggers a highly aggressive and transplantable precursor B-ALL. Tp53/-Lnk ${ }^{-/-}$B-ALLs displayed similar gene expression profiles to human Ph-like B-ALLs, supporting use

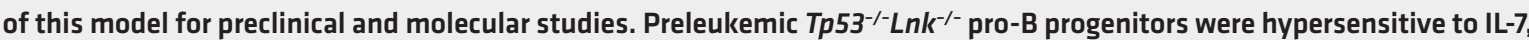
exhibited marked self-renewal in vitro and in vivo, and were able to initiate B-ALL in transplant recipients. Mechanistically, we demonstrated that LNK regulates pro-B progenitor homeostasis by attenuating IL-7-stimuated JAK/STAT5 signaling via a direct interaction with phosphorylated JAK3. Moreover, JAK inhibitors were effective in prolonging survival of mice

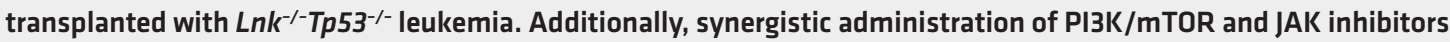
further abrogated leukemia development. Hence, our results suggest that LNK suppresses IL-7R/JAK/STAT signaling to restrict pro-/pre-B progenitor expansion and leukemia development, providing a pathogenic mechanism and a potential therapeutic approach for B-ALLs with LNK mutations.
\end{abstract}

\section{Introduction}

Acute lymphoblastic leukemia (ALL) is the leading cause of cancer-related death in young people and commonly has a poor outcome in adults. Gene profiling and exome sequencing of clinically high-risk ALLs have led to the recent identification of the Philadelphia chromosome-like (Ph-like) ALL subtype (1-3). This subtype exhibits a gene expression profile similar to Ph-positive ALL and exhibits similar clinical behavior to Ph-positive ALL, but it lacks a $B C R-A B L 1$ rearrangement. The Ph-like ALL subgroup comprises $10 \%-15 \%$ of pediatric ALL and is thus 3-4 times more common than Ph-positive ALL in children $(4,5)$. A recent large-scale genomic analysis on 1,725 patients with B cell precursor ALL (BCP-ALL) found that the prevalence of Ph-like ALL increases with age, from $10 \%$ among children with standard-risk ALL to $27 \%$ among young adults with ALL, and is associated with a poor outcome (6). Children and adults with Ph-like ALL have an extremely high risk of relapse and poor survival when treated with conventional chemotherapy.

Three major signaling pathways are found perturbed in high-risk ALLs, the TP53/RB pathway predominantly involving deletions of $C D K N 2 A / C D K N 2 B$, loss of function mutations/ deletions of lymphoid transcription factors IKZF1 and PAX5, and tyrosine kinase activation lesions $(6,7)$. Most Ph-like ALLassociated mutations/deletions/translocations identified to date

Conflict of interest: The authors have declared that no conflict of interest exists. Submitted: February 12, 2015; Accepted: February 3, 2016.

Reference information: / Clin Invest. 2016;126(4):1267-1281. doi:10.1172/JCI81468 are known or predicted to activate oncogenic cytokine receptor signaling, particularly of JAK-associated pathways. Approximately half of Ph-like ALLs have CRLF2 (cytokine receptor-like factor 2) rearrangements resulting in overexpression of CRLF2, many of which have concomitant gain-of-function (GOF) alterations in JAK pathway-associated genes, including JAK1, JAK2, and IL7RA (IL-7 receptor $\alpha$ chain) (2, 8-12). IL-7 binds to IL-7R $\alpha$ and $\gamma c$ chain to activate STAT5. The CRLF2 protein heterodimerizes with the IL-7R $\alpha$ chain to create the thymic stromal lymphopoietin receptor (TSLPR) and binds its ligand, TSLP $(13,14)$. TSLP likely activates STAT5 through JAK2 (15-18). Constitutive activations of STAT5, S6, and/or ERK are induced in murine pro-B lymphoma $\mathrm{Ba} / \mathrm{F} 3$ cell lines transduced with human ALLspecific JAK and/or IL7RA or CRLF2 mutations (8, 11, 12, 19-21).

The lymphocyte adaptor protein LNK (also called SH2B3) has emerged as a powerful and important negative regulator of cytokine signaling during hematopoiesis and B-lymphopoiesis. Lnk ${ }^{-1-}$ mice exhibit a 3- to 5-fold elevation in wbc (22), increased pre-/proand immature B cells in the BM (23), and a marked expansion in the hematopoietic stem cell (HSC) pool $(24,25)$. Mechanistically, LNK attenuates cytokine signaling in multiple hematopoietic lineages. In the HSC compartment, LNK constrains HSC self-renewal largely through inhibition of thrombopoietin (TPO) and it's receptor myeloproliferative leukemia protein-mediated (MPL/CD110-mediated) JAK2 activation (25-27). We previously reported that LNK directly interacts with JAK2 and that LNK deficiency potentiates JAK2 activation in HSCs (26). In the B cell 
Table 1. LNK synergizes with TP53 in suppressing B-ALL development in mice

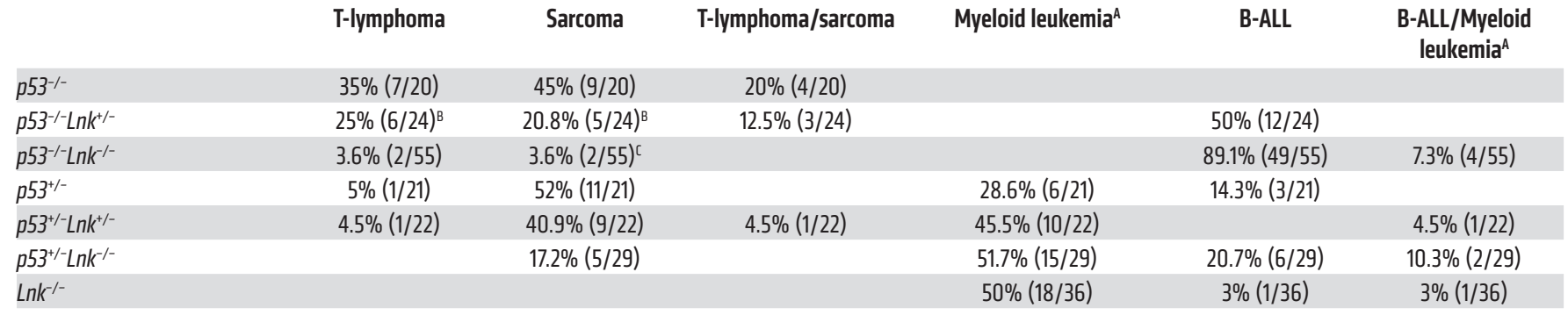

Tumor spectrum and frequencies in $p 53 /$ Lnk mutant mice are shown. Frequency of the tumor incidence is shown in the table, while the number of positive mice/total number of mice examined is shown in parentheses. ${ }^{A}$ Myeloid leukemia includes monocytic tumors. ${ }^{B}$ One mouse also had B-ALL. 'Both mice had sarcoma and B-ALL.

lineage, LNK is thought to dampen stem cell factor (SCF) and its receptor c-KIT, IL-7/IL-7R, and FLT3 function $(22,23,28)$; however, LNK-mediated signaling in B cells has not been fully examined. Neither is it known which JAK family members are regulated by LNK in lymphoid cells. Recently, somatic deletion/mutations in $L N K$ (also known as SH2B3) were found in various B-ALLs and T-ALLs in children $(2,6,29,30)$. A recent sequencing effort identified kinase-activating alterations in $91 \%$ of Ph-like ALL patients. Chromosomal rearrangements involving $A B L, J A K 2$, and CRLF2 and sequence mutations involving FLT3, IL7R, or SH2B3 are most common (6). Specifically, 9 of 154 patients of pediatric and young adult Ph-like B-ALLs have mutations in SH2B3 (5.8\%) (6). Furthermore, genetic loss of $L N K$ contributes to pediatric ALL development (30), underscoring the importance of LNK in ALLs. However, the mechanisms by which it impacts ALL initiation or progression remain poorly understood.

In this report, we investigated mechanisms by which LNK regulates normal B cell and B-ALL development. We developed a B-ALL mouse model that resembles human Ph-like B-ALLs in a gene expression signature. Mechanistically, we found that LNK suppresses normal B progenitor cell and B-ALL development through downregulating IL-7-mediated JAK/STAT signaling. We also explored therapeutic strategies using this mouse model of B-ALL. Together, our studies enhance our understanding of Ph-like B-ALLs and provide insight into therapeutic strategies.

\section{Results}

LNK synergizes with TP53 and INK4A/ARF in suppressing B-ALL development in mice. We previously reported that $\mathrm{Lnk}^{-/-}$mice spontaneously develop chronic myelogenous leukemia-like (CML-like) myeloproliferative neoplasms (MPNs) upon aging (31). Furthermore, following the natural progression of these mice, we found that most $\mathrm{Lnk}^{-/-}$mice died of monocytic tumors around 1.5 years, while about $5 \%$ developed B-ALL at 2 years of age (Tables 1 and 2). $\mathrm{Lnk}^{-/-}$mice develop B-ALL at low penetrance and long latency, suggesting the need for cooperating events. To explore genetic interactions that could cooperate with Lnk deficiency in the development of ALL, we generated mice harboring mutations for both Lnk and tumor suppressors Tp53 or Ink4a/Arf. TP53 is associated with poor diagnosis in many subtypes of B-ALL $(32,33)$. The human gene locus that encodes INK4a/ARF,
CDKN2A/CDKN2B, is highly mutated in Ph-like ALL, a subtype of high-risk ALL with poor prognosis $(6,7)$. We found that loss of 1 or 2 alleles of $L n k$ increased cancer-associated mortality in a gene dose-dependent manner when placed on Tp53 heterozygous or homozygous background, respectively (Figure 1A, with the statistical analysis shown in Supplemental Table 1; supplemental material available online with this article; doi:10.1172/ JCI81468DS1). Notably, Tp53 ${ }^{-/} L n k^{-/}$mice died of B-ALL at almost $100 \%$ penetrance by 4 months, while mice deficient for Tp53 alone died of T-lymphoma or sarcoma after 6 months of age, as previously reported (refs. 34-36, Figure 1C, and Table 1). Similarly, Lnk deficiency exacerbated cancer-associated mortality on Ink $4 a /$ Arf heterozygous or homozygous background (Figure 1B, with the statistical analysis shown in Supplemental Table 2) and markedly increased the incidence of B-ALL in Ink4a/Arf $\mathrm{Lnk}^{-1-}$ mice (Figure 1D and Table 2). The B-ALL blasts filled the BM cavity and peripheral blood (PB) and infiltrated into spleen, lymph nodes, thymus, and-in some mice - liver, lung, and kidney (Figure $1 \mathrm{E}$ and data not shown). Further phenotyping of $T p 53^{-/-} \mathrm{Lnk}^{-/-}$ and Ink4a/Arf ${ }^{/-} \mathrm{Lnk}^{-/-}$B-ALL cells revealed that they were precursor B-ALLs as determined by cell surface expression for $\mathrm{B}$ progenitor markers, $\mathrm{CD} 19^{+} \mathrm{IgM}^{-} \mathrm{CD} 43^{+} \mathrm{IL}-7 \mathrm{R} \alpha^{+} \mathrm{AA} 4.1^{+} \mathrm{Kit}^{+/-}$, and aberrant downregulation of B220 (Figure 2A and Supplemental Figure 1). We transplanted a graded dose of sorted B-ALL blasts into sublethally irradiated mice, and the results showed that mice transplanted with as low as 300 B-ALL blasts succumbed in 1 month, indicating that $T p 53^{-/-} \mathrm{Lnk}^{-/-}$and Ink4a/Arf ${ }^{/-} \mathrm{Lnk}^{-/-}$ B-ALLs are highly aggressive and transplantable malignancies (Figure 2, B and D), with leukemia-initiating cells at a frequency about approximately 1 in 200 (Figure 2, C and E). Our data thus establish that LNK synergizes with tumor suppressor TP53 and INK4A/ARF in suppressing B-ALL development in mice.

Lnk loss of heterozygosity in B-ALLs. It is important to note that about half of Tp53/- Lnk ${ }^{+/-}$mice developed B-ALL, while the others developed T-lymphoma (Table 1). To determine Lnk loss-of-heterozygosity ( $\mathrm{LOH}$ ) status in these tumors, we assessed if the B-ALL cells from Tp53/- $L n k^{+/-}$mice had lost the WT allele of Lnk using purified B-ALL cells, along with cells of myeloid, T lineages, and mature $B$ lineages in the same animals. While myeloid and $\mathrm{T}$ cells, as well as normal mature $\mathrm{CD} 19^{+} \mathrm{B} 22 \mathrm{O}^{\text {hi }} \mathrm{B}$ cells, exhibited the germline genotype $T p 53^{-/-} L n k^{+-}$, B blasts were homozy- 
A

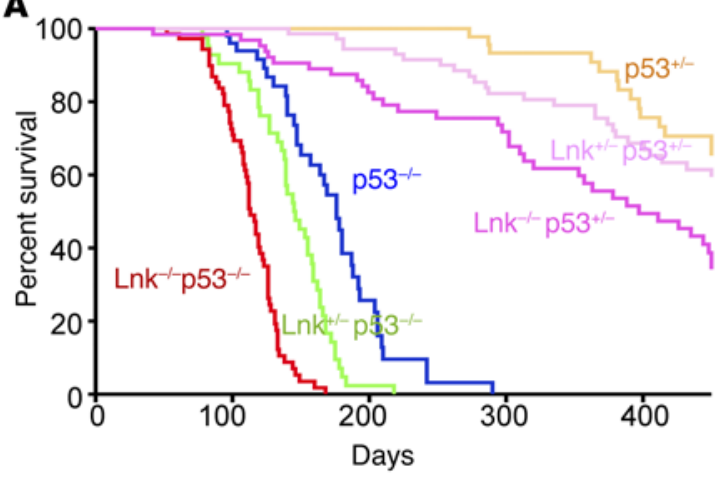

B

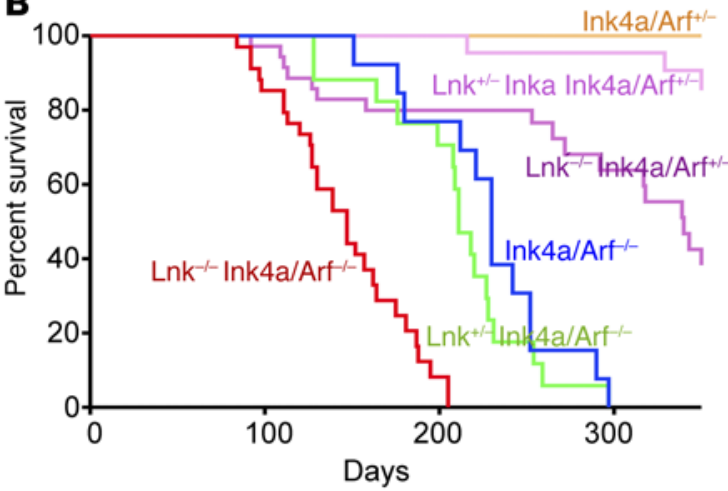

E

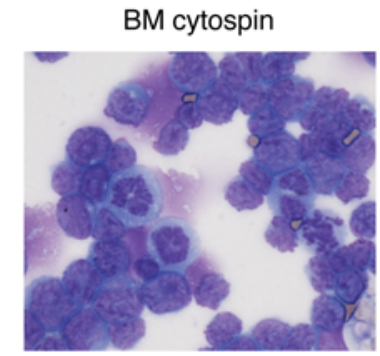

C

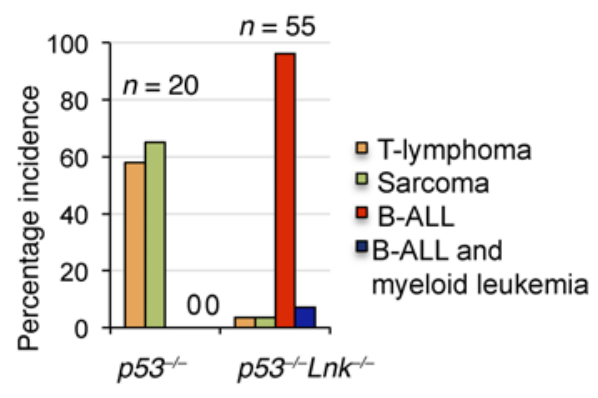

D

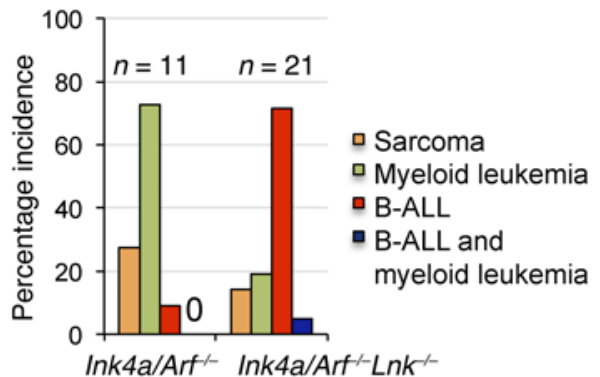

Figure 1. Lnk deficiency synergizes with loss of tumor suppressors TP53 and INIK4A/ARF to promote B-ALL. (A and B)

Kaplan-Meier curves show the survival of $\mathrm{p} 53 / \mathrm{Lnk}(n=$ 42-74 mice per group) (A) or Ink4aArf/Lnk ( $n=12-40$ mice per group) (B) mutant mice. Log-rank $t$ tests are shown in Supplemental Tables 1 and 2. (C and $\mathbf{D})$ The bar graphs depict the tumor incidences of $p 53 /$ Lnk (C) or Ink4aArf/ Lnk (D) mutant mice. $n$ numbers are labeled on top of the bars. (E) Histology of

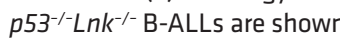
as Wright-Giemsa staining of BM cytospins (left, $\times 600$ magnification), $H \& E$ staining of $B M$ sections (middle, $\times 200$ ), and IHC with anti-B220 antibodies (right, $\times 100$ ). gous for the $L n k$-null alleles (Figure 3A) in 6 of 6 mice examined. In contrast, T-lymphomas from $T p 53^{-/-} \mathrm{Lnk}^{+/-}$mice retained the WT allele of Lnk (Supplemental Figure 2). Our data suggest that Lnk is a bona fide tumor suppressor in restraining B-ALL development. Furthermore, hematopoietic stem and progenitor cells (LSK ${ }^{+}$HSPCs), hematopoietic progenitors (LKS $)$, and common lymphoid progenitors (CLPs) exhibited the germline genotype

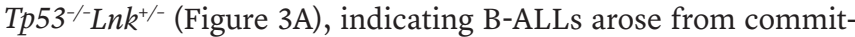
ted $\mathrm{B}$ progenitor cells and, moreover, loss of both alleles of $L n k$ is crucial for fully transforming $B$ progenitors.

Tp53/-Lnk ${ }^{-/-}$pro-B progenitors initiate $B-A L L$. To investigate the mechanisms by which Tp53/Lnk deficiency promotes B-ALL development, we set out to examine BM progenitor cells from young $T p 53^{-/-} \mathrm{Lnk^{-/ }}$ mice before any signs of malignancy, here referred to as preleukemic mice. We isolated LSK cells from young

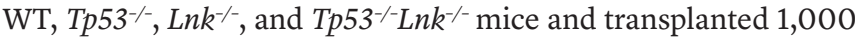
LSKs along with isogenic BM competitors into lethally irradiated recipients. All mice transplanted with preleukemic $\mathrm{Tp} 53^{-/-} \mathrm{Lnk^{-1 }}$ LSK cells succumbed to B-ALL within 3 months, while Tp53-1transplanted mice died significantly later with T-lymphoma (Figure $3 \mathrm{~B})$. This result indicates that TP53/LNK suppresses B-ALL development in a cell-autonomous manner. Of note, some mice transplanted with $T p 53^{-/} \mathrm{Lnk}^{-/-}$cells developed B-ALL along with T-lymphoma in the thymus (data not shown), suggesting that Lnk deficiency does not switch tumor types or suppress T-lymphoma; rather, it promotes B-ALL development. We next transplanted purified pro-B cells from preleukemic Tp53/- $L n k^{-/-}$mice and found that, indeed, $\mathrm{Tp} 53^{-/-} \mathrm{Lnk}^{-/-}$pro-B progenitors were able to initiate B-ALL (Figure 3C).

Tp53/-Lnk ${ }^{-1-}$ B blasts resemble human Ph-like B-ALLs. To determine if $L n k^{-/} p 53^{-/-}$B-ALLs exhibit similar molecular features to human B-ALL, we performed genome-wide expression profiling. We compared $L n k^{-/-} p 53^{-/-}$B blasts to pro-B/pre-B (B220+Ig$\mathrm{M}^{-} \mathrm{Nk} 1.1^{-} \mathrm{Ly}_{6 \mathrm{C}}{ }^{-} \mathrm{CD} 43^{+} \mathrm{AA} 4.1^{+} \mathrm{CD} 19^{+}$) cellsisolated from WT, $\mathrm{Lnk}^{-/}$, p53\%-, and preleukemic $L n k^{-/-} p 53^{-/-}$mice. Remarkably, gene set enrichment analysis (GSEA) (37) revealed that $L n k^{-/-} p 53^{---}$B blasts specifically exhibit gene signatures of human Ph-like B-ALL (ref. 6 and Figure 3D) and R8 subgroup of B-ALL with poor prognosis (3) but not other R1-R7 types (data not shown). Thus, this mouse line represents a preclinical model for human B-ALLs with active kinase signatures, which is characteristic of Ph-like high-risk B-ALL (6). 
Table 2. LNK synergizes with INK4a/Arf in suppressing B-ALL development in mice

\begin{tabular}{|c|c|c|c|c|}
\hline & Sarcoma & Myeloid leukemia ${ }^{A}$ & B-ALL & B-ALL/ Myeloid leukemia ${ }^{A}$ \\
\hline Ink4a/Arf ${ }^{--}$ & $27.3 \%(3 / 11)$ & $72.7 \%(8 / 11)^{\mathrm{B}}$ & $9.1 \%(1 / 11)$ & \\
\hline Ink4a/Arf $f^{/-} L n k^{-1-}$ & $14.3 \%(3 / 21)$ & $19 \%(4 / 21)^{\mathrm{B}}$ & $66.7 \%(14 / 21)$ & $4.8 \%(1 / 21)$ \\
\hline Ink4a/Arf'/-Lnk ${ }^{\prime-}$ & $5.3 \%(1 / 19)$ & $68.4 \%(13 / 19)^{c}$ & $10.5 \%(2 / 19)$ & $15.8 \%(3 / 19)$ \\
\hline $\operatorname{Lnk}^{-1-}$ & & $50 \%(18 / 36)$ & $3 \%(1 / 36)$ & $3 \%(1 / 36)$ \\
\hline
\end{tabular}

Tumor spectrum and frequencies in Ink4aArf/Lnk mutant mice are shown. Frequency of the tumor incidence is shown in the table, while the number of positive mice/total number of mice examined is shown in parentheses. ${ }^{A}$ Myeloid leukemia includes monocytic tumors. ${ }^{B}$ One mouse also had sarcoma. ${ }^{\mathrm{C} O n e}$ mouse also had T-lymphoma. Since Ink4a/Arf/- mice survive over 400 days, we do not have enough mouse numbers to quantify tumor spectrum in these mice.

LNK controls normal B cell and B-ALL development independently of the Mpl pathway or its effects in HSCs. We and others previously established that LNK regulates HSC homeostasis and self-renewal largely through limiting the TPO/MPL/JAK2 pathway (25-27). To determine if LNK plays a cell-autonomous role in B cells or if the B cell expansion is secondary to the HSC phenotype, we examined B cell development in $\mathrm{Mpl}^{-/-} \mathrm{Lnk}^{-/-}$mice. We found that $\mathrm{Mpl}^{\mathrm{H}^{-/}} \mathrm{Lnk}^{-/-}$ mice exhibited reduced HSPC number (Figure 4A), similar to $\mathrm{Mpl}^{-/-}$mice as we reported previously (26). In contrast, $\mathrm{Mpl}^{\mathrm{H}^{--}} \mathrm{Lnk}^{-/-}$ mice showed an expansion in B cells similarly to that in $\mathrm{Lnk}^{-/-}$mice (Figure 4B). Moreover, Tp53-- $\mathrm{Mpl}^{-/-} \mathrm{Lnk}^{-/-}$triple-nullizygous mice developed B-ALL around 3 months with $100 \%$ penetrance and, interestingly, faster than $T p 53^{-/-} \mathrm{Lnk}^{-/}$double-nullizygous mice (Figure 4C), indicating that LNK controls normal B cell and B-ALL development independently of the MPL/JAK2 pathway or its effects in HSCs.

$L N K$ regulates pro- $B$ and pre- $B$ homeostasis. We thus focused our study on the role of LNK in committed lymphoid progenitors, in particular B progenitors. We determined the numbers of lymphoid-primed multipotential progenitors (LMPP) (38), CLPs (39), pre-pro-B progenitors, pro-B, and pre-B cells in WT and $\mathrm{Lnk}^{-/}$mice at both young and old ages using well-established cell surface markers by flow cytometry $(40,41)$. Our results suggest that Lnk deficiency did not affect LMPP, CLP, or pre-/pro-B progenitor numbers at the young age, nor did it affect the decline of these early lymphoid progenitors upon aging (Figure $5 \mathrm{~A}$ and Supplemental Figure 3). Rather, pro-B cells were the earliest B progenitor stage elevated in $\mathrm{Lnk}^{-/-}$mice, and cells of later developmental stages - e.g., pre-B and immature B cells - were also correspondingly increased in the BM (Figure 5, B and C). Importantly, whereas WT pro-B cells declined upon aging (40, 42), $\mathrm{Lnk}^{-/-}$pro-B cells accumulated (Figure $5 \mathrm{~B}$ ), suggesting LNK also regulates pro-B cell aging. Moreover, we found that pro-B cells in young preleukemic $\mathrm{Tp} 53^{-/-} \mathrm{Lnk}^{-/-}$mice were markedly increased in comparison with WT and single KO mice (Figure 5D). Thus, LNK regulates pro-B cell homeostasis and aging, and synergistic loss of $L n k$ and Tp53 leads to a further expansion of pro-B progenitors.

Lnk/Tp53 deficiency promotes B progenitor cell self-renewal and superior responses to $I L-7$. Since $T p 53^{-/}-L n k^{-/-}$B blasts bear surface markers resembling pro-and pre-B progenitors, we next assessed the self-renewal ability of pro-B cells $\left(\mathrm{CD} 19^{+} \mathrm{B} 22 \mathrm{O}^{\mathrm{lo}} \mathrm{IgM}^{-} \mathrm{CD} 43^{+} \mathrm{AA} 4.1^{+}\right)$ using serial replating assays. While WT or $L_{n k^{-/-}}$pro-B cells did not replate in the presence of IL-7 and SCF, Tp53-- and preleukemic $T p 53^{-1-} \mathrm{Lnk}^{-/-}$pro-B cells replated for over 5 rounds (Figure $6 \mathrm{~A}$ ). Importantly, $\mathrm{Tp}^{\mathrm{N}} 53^{--} \mathrm{Lnk^{-- }}$ pro-B cells showed enhanced sensitivity to IL-7 compared with $T p 53^{-/-}$cells in generating increased numbers of colonies (Figure 6B) and colonies with bigger sizes (Figure 6C). In liquid culture, $T p 53^{-/-} \mathrm{Lnk}^{-/}$pro-B cells expanded at a much faster rate and for a more extended period of time compared with $T p 53^{-/-}$ cells or cells of all other genotypes (Figure 6D). We further assessed pro-B progenitor proliferation and differentiation in vivo using adoptive transfers. At 3 weeks after BMT, we found that $T p 53^{-/-} \mathrm{Lnk^{-/ }}$ pro-B cells gave rise to markedly higher $\mathrm{B}$ cell reconstitution in host spleens compared with WT or single KO mice (Figure 6E), indicating that synergistic loss of Lnk and Tp53 enhances B cell expansion in vivo. Thus, our data suggested that Tp53/Lnk deficiency promotes B progenitor self-renewal and superior responses to IL-7.

Lnk deficiency enhances STAT5 activation in response to $I L-7$. IL-7 is the primary cytokine regulating pro-B cell expansion. We thus set out to investigate the role of LNK in regulating IL-7 signaling in $\mathrm{B}$ progenitors. We first sorted $\mathrm{CD} 19^{+} \mathrm{B} 22 \mathrm{O}^{\text {lo }} \mathrm{B}$ precursor cells from the BM, starved them, and subsequently subjected them to graded concentrations of IL-7 followed by Western blot (WB) analysis (Supplemental Figure 4A). $L n k^{-/-}$B cells showed increased IL-7 sensitivity in activating JAK1/JAK3 and STAT5 phosphorylation when compared with WT or Tp53/- cells; of note, preleukemic Tp53-- $\mathrm{Lnk}^{-/-}$double-KO B cells exhibited more pronounced effects in promoting IL-7 signaling than $\mathrm{Lnk}^{-/}$singleKO (Supplemental Figure 4A). Since the CD19 ${ }^{+} B 220^{\text {lo }}$ population contains a mixture of pro-, pre-, and immature B cells, we developed a phospho-flow cytometric strategy that allows us to differentiate and study signal transduction simultaneously in B progenitor cells of different developmental stages. Using this approach, we found that combined staining of cytokines stimulated total BM cells for surface B220 markers and intracellular $\mu$-heavy chain $(\mathrm{IC} \mu)$, together with phospho-specific antibodies, enables separation of subpopulations of B progenitors with distinct IL-7 responsiveness (Figure 7, A-D, and Supplemental Figure $4 \mathrm{~B}$ ). As expected, B220 ${ }^{\text {hi }} \mathrm{IC} \mu^{\text {hi }}$ mature B cells did not show STAT5 phosphorylation (pSTAT5) with IL-7 stimulation, and B220 ${ }^{\text {lo }} \mathrm{IC} \mu^{\text {hi }}$ immature B cells showed very little response to IL-7. While $\mathrm{B} 22 \mathrm{O}^{\mathrm{lo}} \mathrm{IC} \mu^{\text {med }}$ pre-B cells moderately increased pSTAT5, $\mathrm{B} 22 \mathrm{O}^{\mathrm{lo}} \mathrm{IC} \mu^{-}$pro-B cells exhibited the greatest response among all populations to IL-7 in activating pSTAT5 (Figure 7, A-D). Impor- 

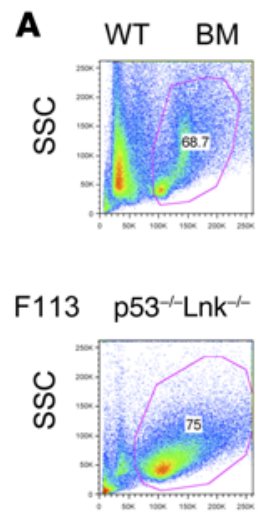

FSC
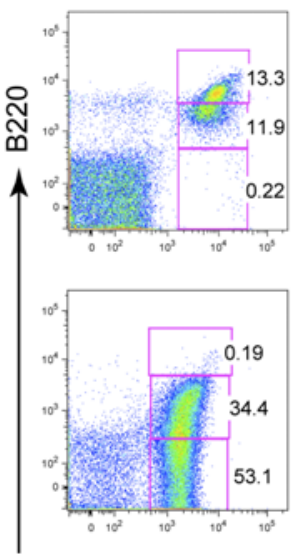

CD19
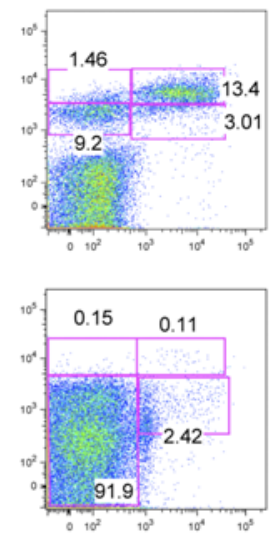

$\lg \mathrm{M}$
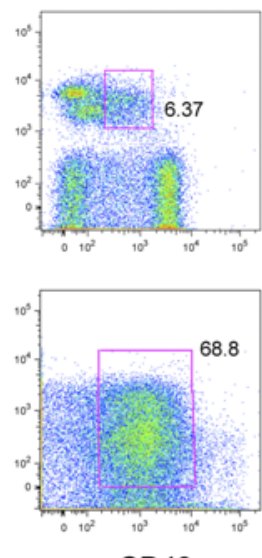

CD43
B209

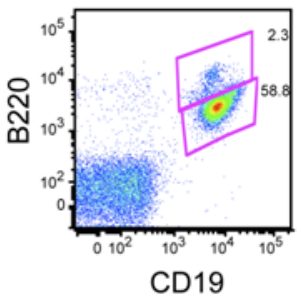

Ink4a/Arf ${ }^{-1} \mathrm{Lnk}^{-1-}$

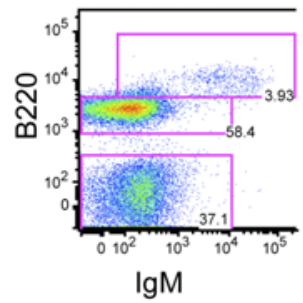

B
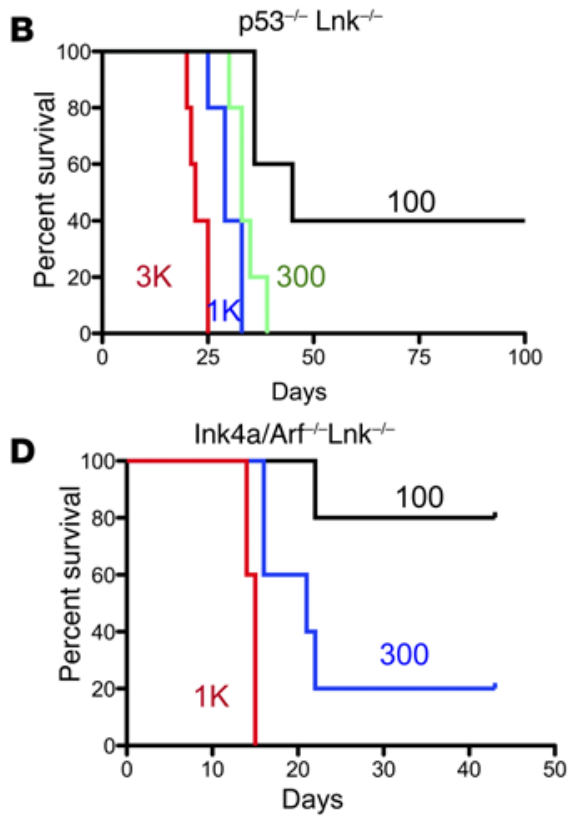

B361
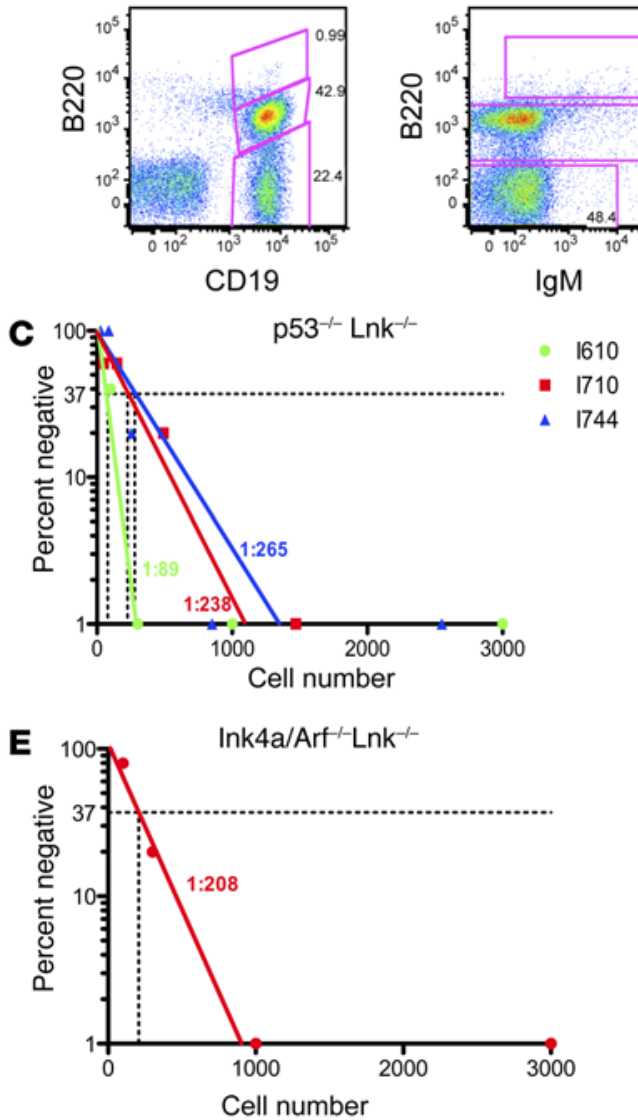

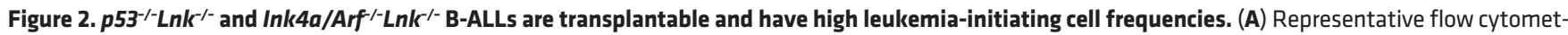

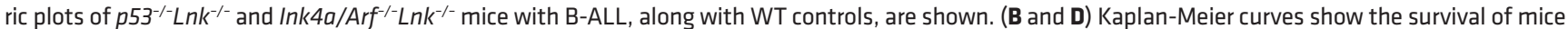

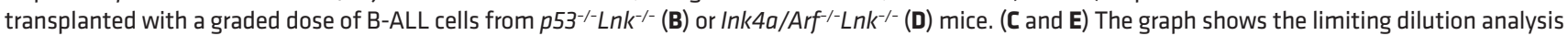

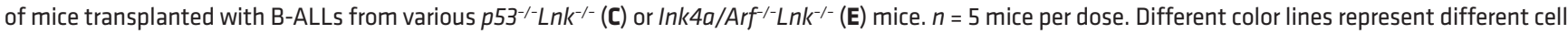
dosages injected in $\mathbf{B}$ and $\mathbf{D}$. Different color lines represent different donor mice in $\mathbf{C}$ and $\mathbf{E}$. The black horizontal dashed lines indicate $37 \%$ negative points, and the vertical lines that intersect with the horizontal lines mark the frequency. Estimated leukemia-initiating cell frequencies are shown for each donor.

tantly, $L n k^{-/}$, but not $p 53^{-/}$, pro-B cells exhibited enhanced response to IL-7 in activating STAT5 (56\% vs. $35 \%)$, indicating Lnk deficiency promotes IL-7-mediated JAK/STAT signaling (Figure 7, B and C). Preleukemic $L n k^{-/}-p 53^{--}$pro-B cells showed similar percentage of $\mathrm{pSTAT}^{+}$cells compared with $\mathrm{Lnk}^{-/}$pro-B, attesting the importance of Lnk-controlled JAK/STAT signaling in B progenitor homeostasis. While the preleukemic $L n k^{-/} p 53^{-/-}$ pro-B cells displayed slightly increased pSTAT5 intensity, the leukemic blasts showed markedly increased pSTAT5 upon IL-7 stimulation (Figure 7, B and D). This is manifested at both the 
A
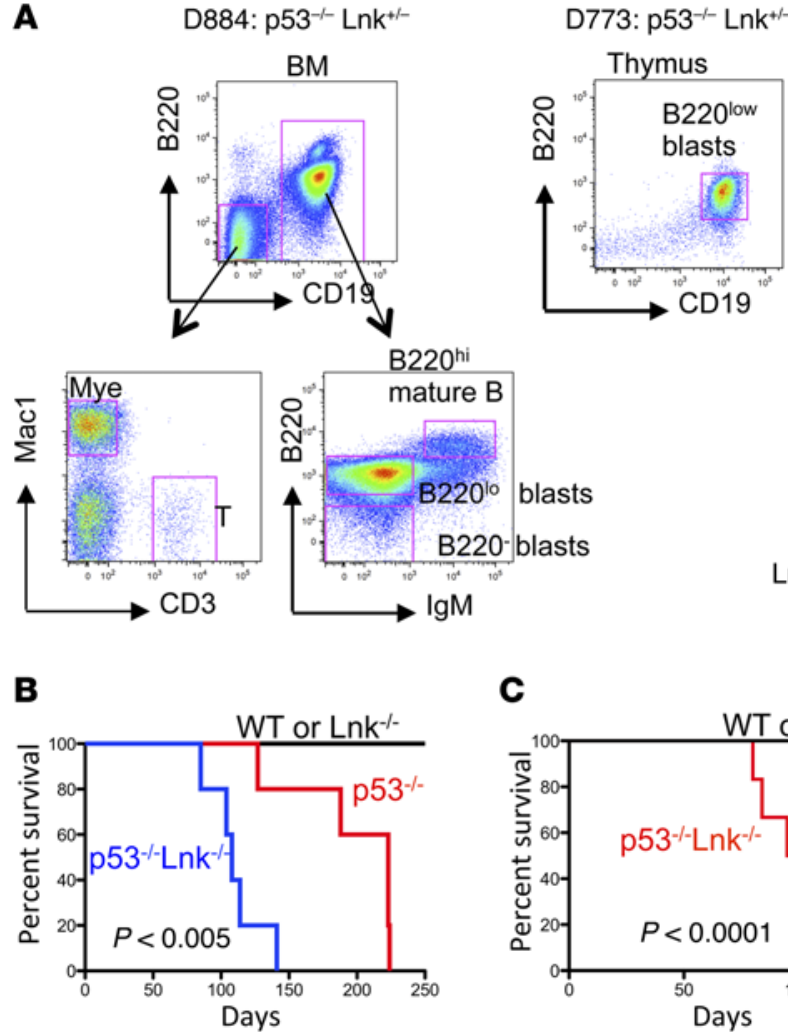

C

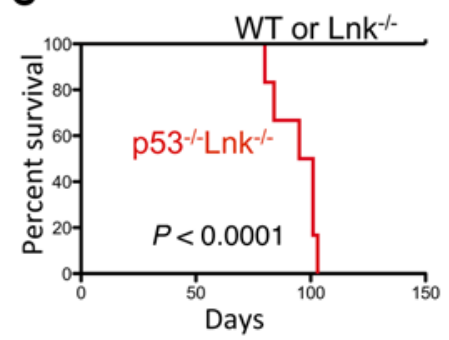

D884

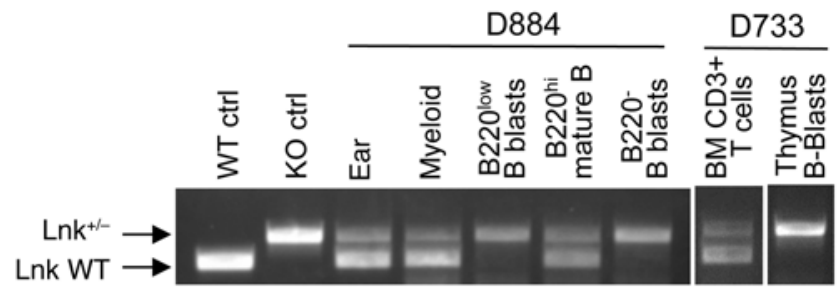

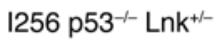

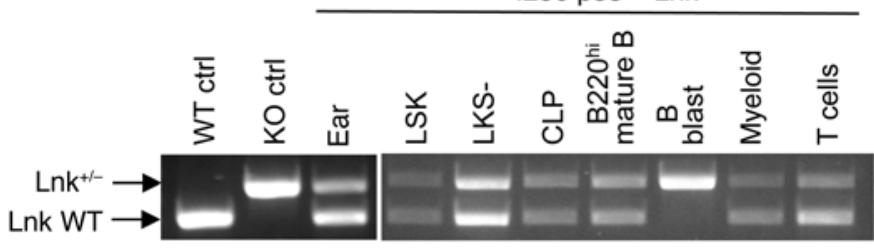

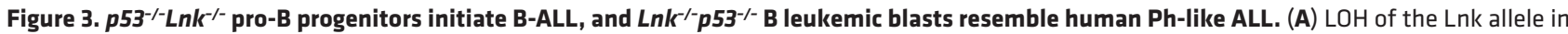

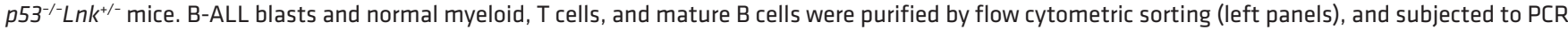

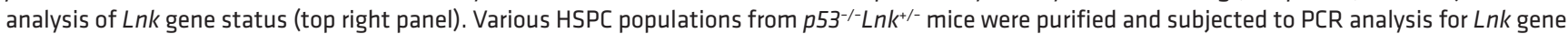

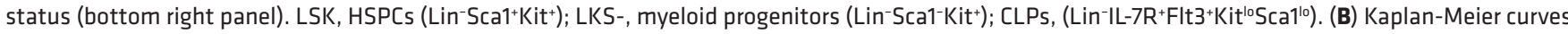

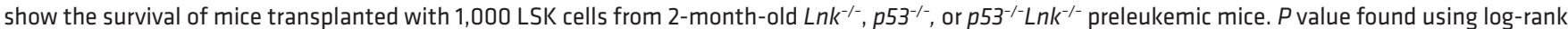
$t$ test. $n=5$ mice per group. (C) Kaplan-Meier curves show the survival of mice transplanted with 100,000 pro-B cells from young preleukemic $p 53^{-1-L n k^{-/-}}$ mice. $P$ value found using log-rank $t$ test. $n=6$ mice per group. (D) Enrichment plots of CSEA analysis using $L n k^{-/} p 53^{-/-}$B leukemic blasts (4 samples) versus control B progenitors (11 samples consist of $3 \mathrm{WT}, 3 \mathrm{Lnk}^{-1-}, 3 \mathrm{p} 53^{-1-}$, and 2 preleukemic $p 53^{-1-L n k^{-1}}$ mice) express data against Ph-like B-ALL gene signature from the previous publication (6). NES, normalized enrichment score; FDR, false discovery rate.

level of amplitude (mean fluorescence intensity [MFI]) and the percentage of cells responded. Of note, we found that preleukemic $L n k^{-1} p 53^{-1-}$ B progenitors showed a slight increase, and leukemic blasts displayed a marked elevation in basal pSTAT5 activation (Figure 7E). Consistently, Leukemic $\mathrm{Lnk}^{-/-} \mathrm{p} 53^{-/-}$blasts showed both basal and IL-7-induced activation of JAK1 and JAK3 but not JAK2 (Figure 7F). Altogether, our data indicate that the synergistic effects of Lnk and $p 53$ loss result in enhanced IL-7/ JAK/STAT signaling and B progenitor cell transformation.

LNK interacts with phosphorylated JAK3. We previously reported that the LNK SH2 domain directly interacts with phosphorylated JAK2 (pY813) upon TPO stimulation in HSPCs (26); however, how LNK interacts with the lymphoid signaling pathway has not been explored. Neither was it known which JAK family members were regulated by LNK in the IL-7R pathway, since IL-7R activates JAK1/3 but not JAK2. We performed an unbiased proteomic screen to identify the peptide motifs with which the LNK SH2 domain interacted (Supplemental Table 3). The consensus of the LNK SH2 domain binding motif is $\operatorname{xxpY}(\mathrm{V} / \mathrm{I} / \mathrm{E}) \mathrm{xL}$ (Supplemental Table 3 and Supplemental Figure 5). Of note, the LNK SH2 has some preference for a Pro at pY-2 and an Asp at pY-1 positions. The preference for an Asp at pY-1 is reinforced by the almost total absence of Arg or Lys at the same position. The JAK2 (Y813) and JAK3 motifs (Y785) are PDpYELL and SDpYELL, respectively, which are good matches to the consensus. Next, we used LNK to pull down WT, kinase-inactive JAK3 mutant Y785F, or the Y980/981F mutant as a control. We found that LNK interacted with phosphorylated JAK3 but not kinase-inactive mutant Y785F and, importantly, LNK itself was tyrosine phosphorylated by JAK3 (Figure 7G). JAK1 lacks the LNK consensus motif and was not able to pull down phosphorylated LNK (data not shown). To investigate the effects of LNK overexpression in IL-7R-mediated growth, we expressed vector alone, WT, or the SH2 domain mutant (R384E) of LNK in the pro-B cell line, BaF3 cells stably expressing IL-7R (Figure 7H). Indeed, WT LNK, but not the SH2 domain mutant, inhibited IL-7-mediated cell growth (Figure 7H). Taken together, our results suggest that LNK is a negative regulator of IL-7R-mediated JAK/SAT signaling in B progenitors through a direct interaction between LNK and JAK3.

JAK inhibitors are effective in vivo and against $\mathrm{Lnk}^{-1-} \mathrm{p} 53^{--}$leukemic blasts. Our signaling studies suggest that LNK-regulated IL-7R/JAK/SAT signaling plays important roles in normal and 

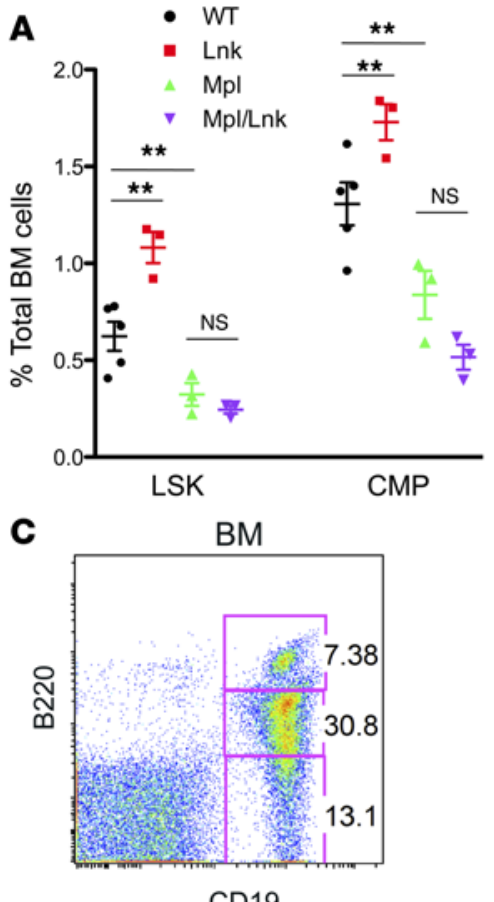

CD19
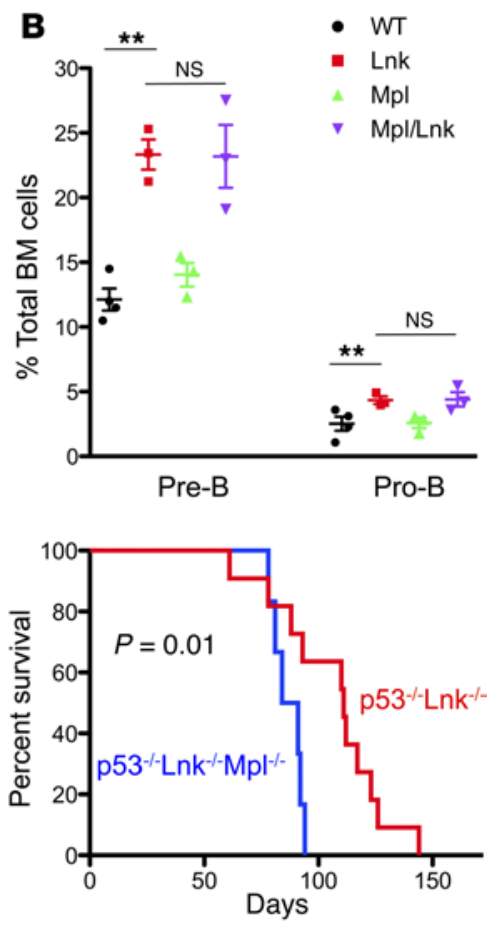

Figure 4. LNK controls normal B cell and B-ALL development independently of $\mathrm{Mpl}$ pathway or its effects on HSCs. (A) $\mathrm{Lnk}^{-/-} \mathrm{Mpl}^{-/-}$double-null mice show decreased HSPC and myeloid progenitor compartments similar to $\mathrm{Mpl}^{-/-}$mice. LSK, $\mathrm{Lin}^{-} \mathrm{Sca1}^{+} \mathrm{Kit}^{+}$cell fraction that contains both HSCs and multipotential progenitors; CMP, common myeloid progenitor, Lin-Sca1-Kit ${ }^{+} \mathrm{CD}_{4}{ }^{+} \mathrm{FcrR}^{-} .{ }^{*} P<0.01$; ns, not significant, 2-tailed Student's $t$ test. $n=3-5$ per group. (B) $\mathrm{Lnk}^{-1-} \mathrm{Mpl}^{-1-}$ double-null mice show increased pre- and pro-B cell frequencies in the BM, similar to $\mathrm{Lnk}^{-1}$ mice. ${ }^{*} P<0.05$; ${ }^{* *} P<0.01$; ns, not significant, 2-tailed Student's $t$ test. $n=3-4$ per group. (C) $p 53^{-1-} \mathrm{Lnk}^{-1-} \mathrm{Mpl}^{-1-}$ triple-null mice develop aggressive B-ALL similarly to

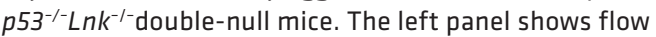
analysis of BM B-ALL cells in $p 53^{-1-} \mathrm{Lnk}^{-1-} \mathrm{Mpl}^{-/-}$mice, and the right panel shows the Kaplan-Meier survival curves of $p 53^{-/-} \mathrm{Lnk}^{-1-}$ and $p 53^{-/-} \mathrm{Lnk}^{-1-} \mathrm{Mpl}^{-/-}$mice. $P$ value found using log-rank $t$ test. $n=6-12$ mice per group. malignant B progenitors, which can be exploited for therapeutic purposes. This finding is corroborated with the results showing that JAK1/2 inhibitor ruxolitinib and JAK3/2 inhibitor tofacitinib inhibited $\mathrm{Lnk}^{-/} \mathrm{p} 53^{-/}$ALL colonogenic growth (Figure 8A). We further treated the mice transplanted with $L n k^{-/} p 53^{---}$B-ALL cells with either vehicle alone or ruxolitinib in vivo. Ruxolitinib significantly delayed leukemia onset and prolonged the survival of transplanted mice (Figure $8 \mathrm{~B}$ ). We then analyzed the mice 10 days after JAK inhibitor treatment and found that JAK inhibition markedly reduced donor leukemia percentage, as well as the spleen weight, in the host spleen (Figure 8, C and D).

JAK and PI3K inhibitors synergistically inhibit Lnk ${ }^{-/-} p 53^{-/-}$leukemic cell growth with in vivo and in vitro. We noted that, under starved condition, $L n k^{-/} p 53^{-/-}$blasts exhibited constitutive activation of AKT, S6, and pERK (Figure 9A), in addition to elevated basal pSTAT5 levels, although IL-7 did not induce pAKT, pS6, or pERK activation in freshly isolated B progenitors in our assay. This result prompted us to investigate if simultaneous inhibition of multiple signaling pathways will further abrogate B-ALL growth. We were able to grow ALL blasts in liquid culture without stromal cell support, which enabled a small-scale combinatorial kinase inhibitor screen for drugs that synergize with JAK inhibition to further abrogate ALL cell growth. We found that PI3K inhibitors, but not MEK inhibitors (selumetinib or trametinib), synergistically abrogated leukemia cell growth with ruxolitinib (Figure 9B and Supplemental Figure 6D). We tested a number of PI3K inhibitors with different specificities against different PI3K isoforms. While inhibitors of p110 (CAL-101 or dactolisib) or p110 $\gamma$ (AS-605240) failed to inhibit ALL cell growth (Supplemental Figure 6D), pan-PI3K inhibitors (PI-103) and PI3K/mTOR dual inhibitors (NVP-BEZ235) synergized with ruxolitinib to abrogate ALL cell growth (Figure 9B and Supplemental Figure 6A). Inhibiting $\mathrm{p} 110 \alpha$ (BYL719) or $\mathrm{p} 110 \beta$ (TGX-221) was also effective, albeit at higher concentrations (Supplemental Figure 6C). Thus, our data indicate the importance of $\mathrm{p} 110 \alpha$ and $\mathrm{p} 110 \beta$ in supporting ALL cell proliferation. WB analysis revealed that PI3K inhibitors inhibited AKT, S6, and 4EBP1 activation, while ruxolitinib inhibited STAT5 and ERK phosphorylation (Figure 9C and Supplemental Figure 6B), thus providing signaling mechanism for their efficacy in precursor B-ALLs.

We next extended our findings to in vivo treatment of B-ALLs. Ruxolitinib or BEZ235, when administrated singly, significantly delayed leukemia onset and prolonged the survival of transplanted mice, while dual inhibition of JAK and PI3K further prolonged the survival (Figure 9D). We then analyzed the mice 10 days after treatments and found that JAK inhibitors and PI3K inhibitors, when administrated singly, reduced donor leukemia percentage in the host BM and spleen as well as the spleen weight (Figure 9, E and F). Dual inhibition of JAK and PI3K markedly abrogated donor leukemia in the host mice (Figure 9, E and F). Taken together, our data implicate that aberrant activation of IL-7R/JAK and PI3K/ AKT signaling pathways by loss of LNK leads to leukemic transformation and the leukemia cells are amenable to JAK inhibitors. Furthermore, targeting JAK/STAT and PI3K/AKT pathways are critical to cripple ALL blast growth.

\section{Discussion}

In this study, we aimed to unravel molecular mechanisms by which LNK controls B progenitor cell development and leukemic transformation and devise effective treatment strategies to eliminate leukemia cells with loss-of-function Lnk mutations. The work presented here provides new insights into LNK function in normal and malignant B progenitor cells. We demonstrated that LNK plays a pivotal role in pro-B homeostasis and aging through regulating IL-7-mediated JAK/STAT signaling. Furthermore, LNK suppresses precursor B-ALL development in mice, and $L n k$ 


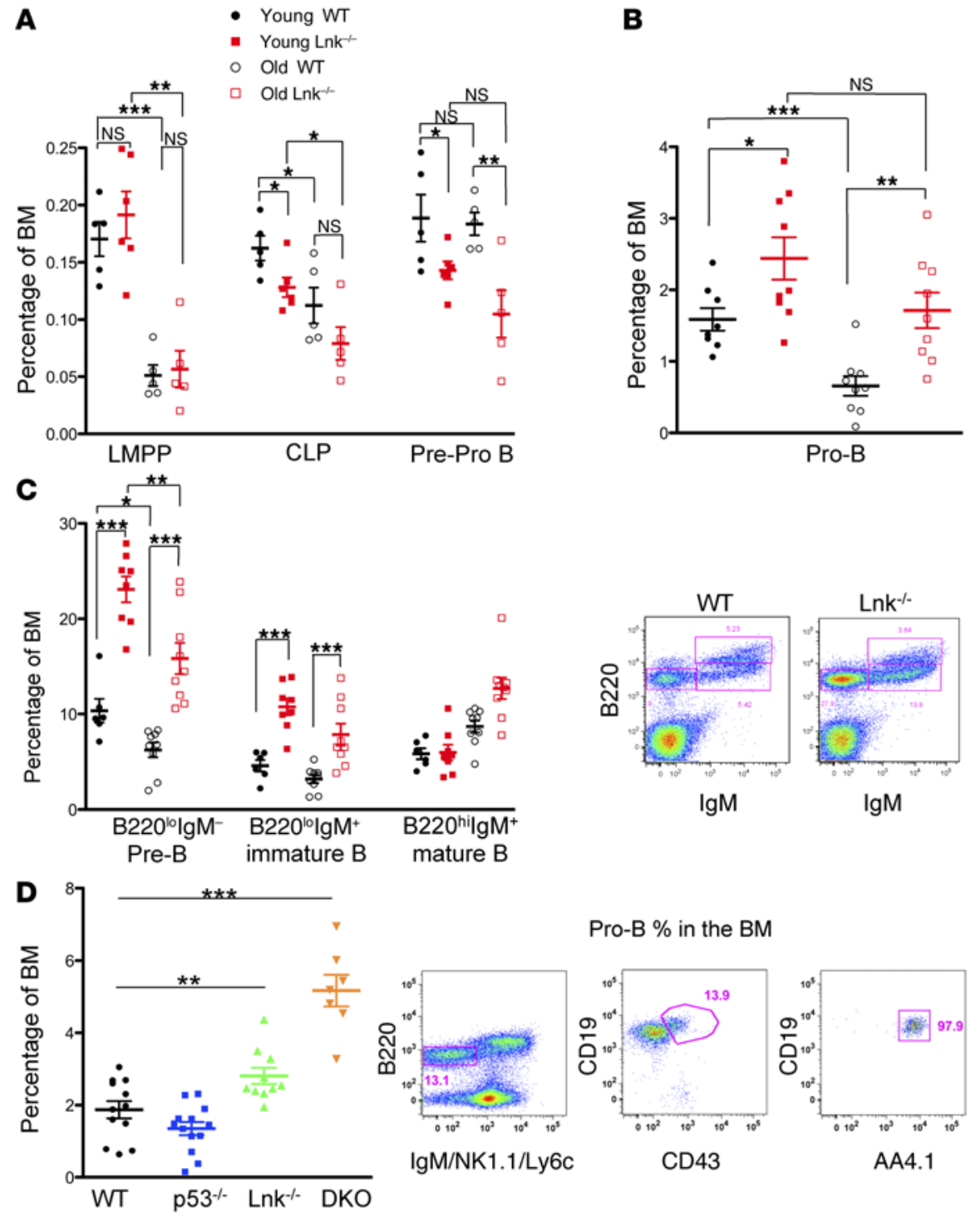

Figure 5. LNK regulates pro-B homeostasis and aging. (A and B) Young and old $\mathrm{Lnk}^{-1-}$ mice show increased pro-B cells in the BM. Percentages of various lymphoid progenitors in young and old mice are shown. The following markers are used: LMPP, $\mathrm{Lin}^{-} \mathrm{Kit}^{+} \mathrm{S} \mathrm{Ca}^{+}{ }^{+} \mathrm{Flt} 3^{\mathrm{hi}}$; CLP, Lin-IL-7R+FIt3+Sca1 ${ }^{10} \mathrm{Kit}^{\mathrm{lo}}$; pre-pro B, Lin-Ly6C-NK1.1-IgM-AA4.1+CD19-B220+; pro-B, B220 ${ }^{+} \mathrm{CD}{ }^{+}$AA4. $1^{+}$CD19 ${ }^{+} .{ }^{*} P<0.05 ;{ }^{*} P<0.01$; ${ }^{* * *} P<0.0001$; ns, not significant, 2-tailed Student's $t$ test. $n=8-9$ mice per group. (C) Young and old $\mathrm{LnK}^{-/-}$mice show increased numbers of pre-B cells and immature B cells in the BM. Representative flow plots are shown. ${ }^{*} P<0.05$; ${ }^{* *} P<0.01$; ${ }^{* *} P<0.001$; ns, not significant, 2-tailed Student's $t$ test. $n=7-9$ mice per group. (D) Young $p 53^{-1-L n k^{-1-} \text { mice }}$ show increased pro-B\% in the BM. Pro-B cells were determined by a stringent panel of mark-

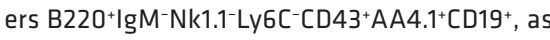
shown by representative flow plots. $n=7-12$ mice per group.

found in $91 \%$ of the cases. Rearrangements involving ABL kinases, JAK2, and cytokine receptors and sequence mutations involving FLT3, IL7RA, or SH2B3 were most common (6). Our results show that LNK and TP53 or INK4A/ARF synergistically suppress B-ALL development in mice, implying that our mouse models are of clinical relevance. The superior responses to IL-7 and the hyperactivation of STAT5 pathway in $L n k^{--} T p 53^{-/}$ B-ALLs are also consistent with the Ph-like high-risk B-ALL phenotype that is associated with dysregulated cytokine signaling. Furthermore, Lnk $^{--T}$ Tp53-- B-ALLs demonstrate a gene expression signature similar to Ph-like B-ALL but not other subtypes, strongly arguing for a role of LNK in this subtype of B-ALL

deficient B-ALLs are sensitive to JAK inhibitors. Our data, along with clinical data showing that $L N K$ (or SH2B3) mutations occur in high-risk ALL patients and are likely associated with aberrant cytokine signaling besides JAK and receptors themselves, suggests that LNK is a bona fide tumor suppressor. Our B-ALL mouse model shows a similar gene expression signature to that of human Ph-like B-ALL, in which $L N K, J A K 2, I L 7 R$, and CRLF2 mutations have been commonly identified, indicating that Lnk-deficient B-ALL mouse model is relevant to this subtype of B-ALL that is associated with poor prognosis. Thus, our studies provide mechanistic and therapeutic insight into the aberrant signaling transduction underlying Ph-like high-risk B-ALLs with LNK mutations.

Recently, a large-scale sequencing effort found that Ph-like precursor B-ALL increases in frequency from 10\% among children to $27 \%$ among young adults with ALL and is associated with a poor outcome (6). Ph-like B-ALLs show aberrations in 3 categories of genes: kinase signaling pathways, lymphoid transcription factors such as IKZF1 and PAX5, and tumor suppressors, namely the INK4A/ARF/TP53/RB pathway $(6,7)$. Kinase-activating alterations are the signature and defining feature of Ph-like ALL, which are with underlying aberrant cytokine signaling. Of note, 5 of 9 patients with $L N K$ mutations also harbor $I L 7 R$ mutations (6). Therefore, our findings showing that LNK interacts with phosphorylated JAK3 that is activated by IL-7R may shed light on the molecular mechanism for this unique clinical connection between LNK and IL-7R.

JAK/STAT signaling pathway aberration is found in over $50 \%$ of Ph-like ALL. Whole-genome or whole-exome sequencing identified patients with mutations in multiple genes within the kinase signaling pathway, with evidence of subclonality in a majority of patients tested (6). Mutations in FLT3, IL7R, JAK1, JAK2, JAK3, NF1, NRAS, and PTPN11 were subclonal, and several cases harbored multiple subclonal mutations in the same pathway, indicating the presence of multiple subclones with distinct mutations activating JAK/STAT and/or RAS signaling. Interestingly, the mutant allele frequency (MAF) of $S H 2 B 3$ is very high at 0.9 , implicating a proliferative advantage of the ALL clone that harbor the mutant $\mathrm{SH} 2 \mathrm{~B} 3$ allele (6). This is consistent with our data showing that LOH in LNK is evident $L n k^{+-} T p 53^{--}$B-ALLs, but not in Tlymphomas. Together, these data suggest a pivotal role for Lnk in the transformation/progression of B-ALL development. 

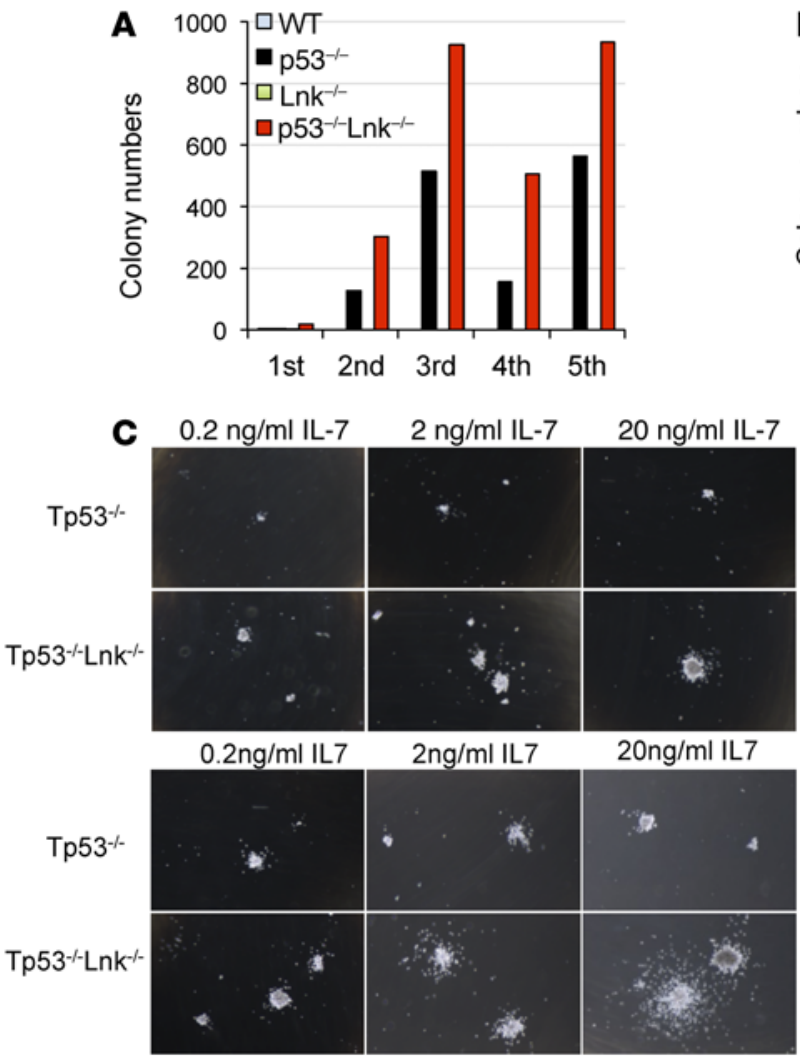

$\mathbf{E}$

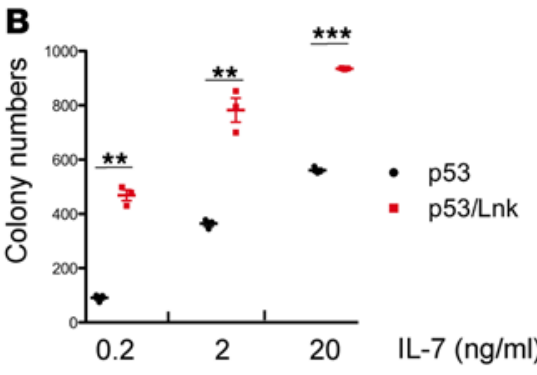

D
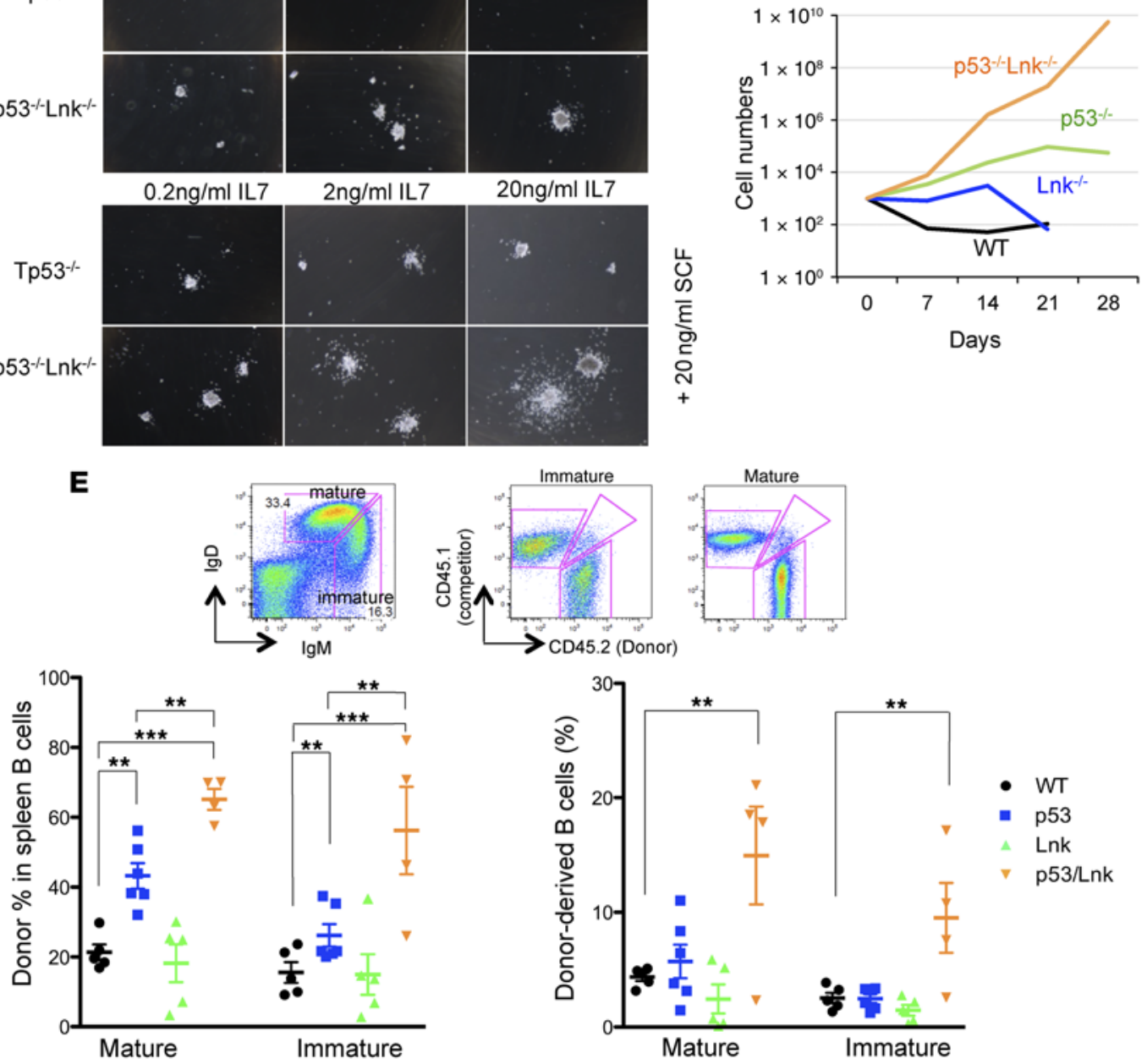

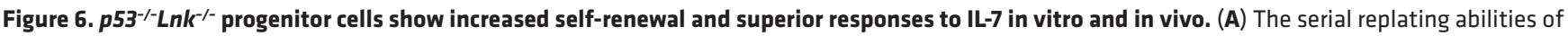
purified pro-B cells from mice of 4 different genotypes in the presence of IL-7 and SCF are shown. Representative graph of 5 independent experiments is shown. (B) The colony formation ability of $p 53^{-1-}$ or $p 53^{-1-} \mathrm{Lnk}^{-1-}$ pro-B cells at the 4 th replating in different concentrations of IL-7 along with $20 \mathrm{ng} / \mathrm{ml} \mathrm{SCF}$ are shown. Representative graph of 4 independent experiments is shown. (C) Photomicrograph of colonies plated in methylcellulose in different cytokine conditions $(\times 40)$. (D) Log growth of purified pro-B cells cultured on OP-9 stromal cells supplemented with IL-7/SCF/FIt3L. Representatives of 4 inde-

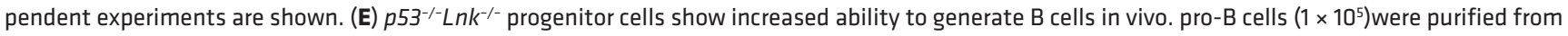
2-month-old WT, $\mathrm{Lnk}^{-/-}, p 53^{-/-}$, or $p 53^{-/-} \mathrm{Lnk}^{-/-}$mice; mixed with $3 \times 10^{5}$ competitor BM cells; and transplanted into lethally irradiated recipients. Chimerisms of donor-derived $\mathrm{IgM}^{+} \lg \mathrm{D}^{+}$mature and $\mathrm{IgM} \mathrm{M}^{-} \mathrm{IgD} \mathrm{D}^{+}$immature $\mathrm{B}$ cells in the spleen of the transplanted mice are shown. $n=4-6$ mice per group. Representative results from 2 independent experiments are shown. ${ }^{* *} P<0.01$; ${ }^{* *} P<0.001,2$-tailed Students' $t$ test. 
A

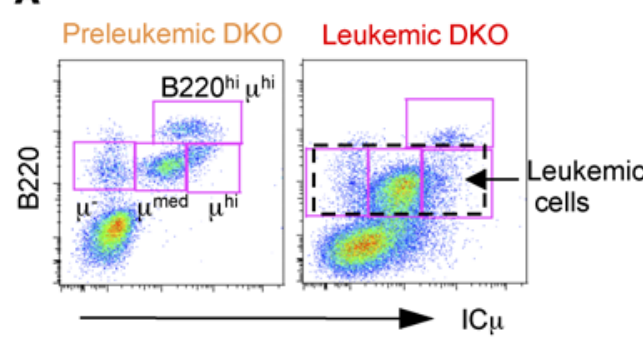

B

C

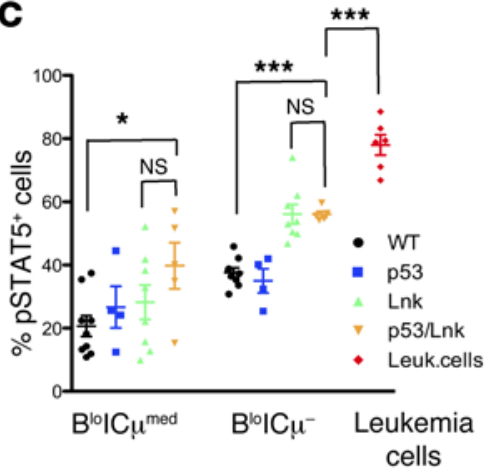

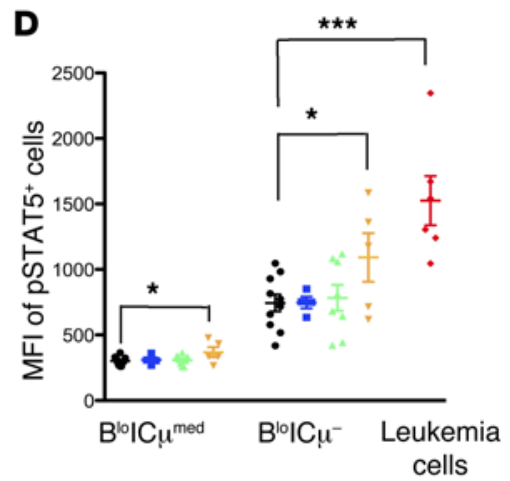

Differentiation

Immature Mature B220 $0^{10} \mid \mathrm{Cu}^{+}$ $\mathrm{B} 220^{\circ} \mathrm{ol} \mathrm{Cu}^{+}$

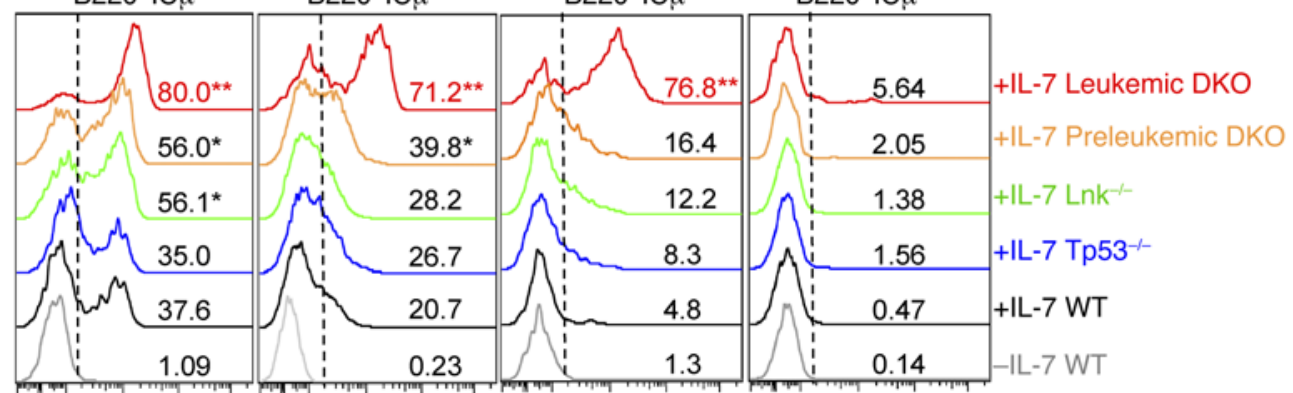

pSTAT5

E
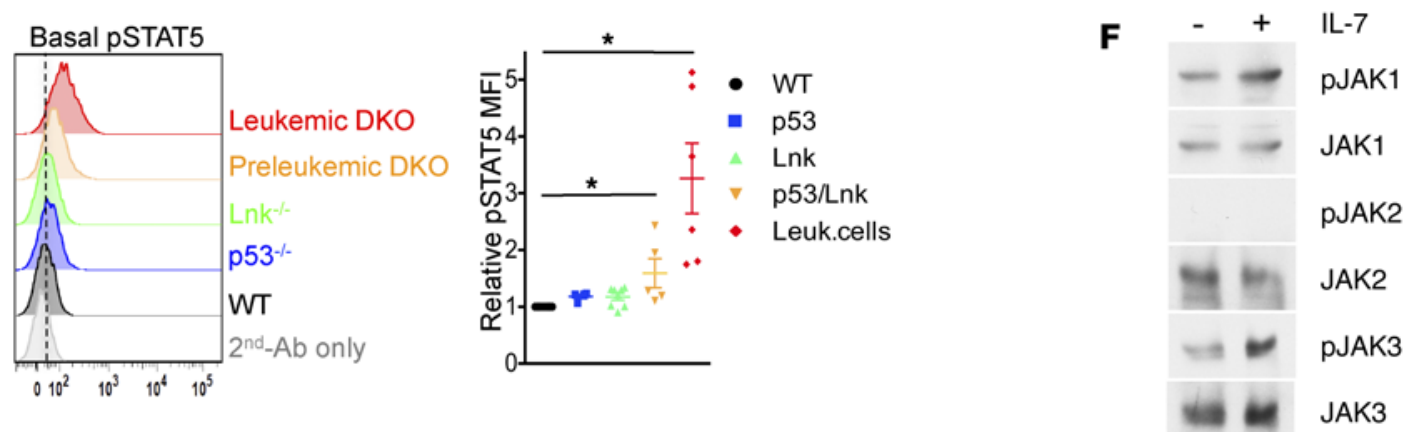

G

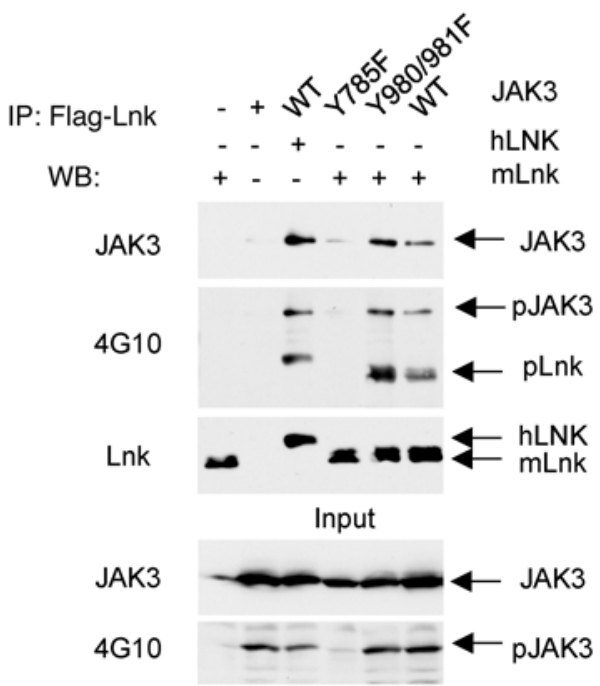

H

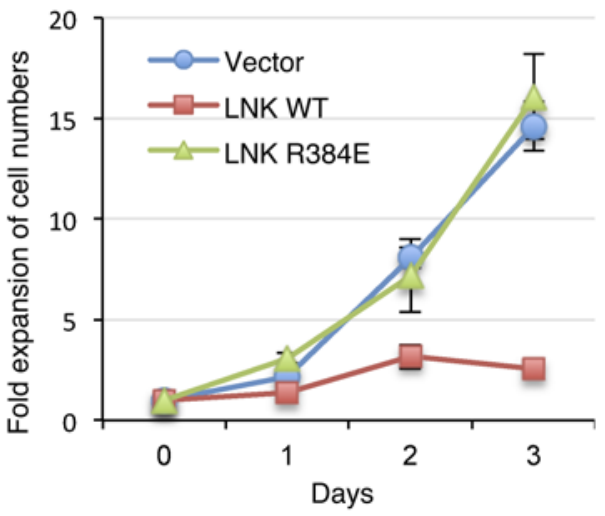




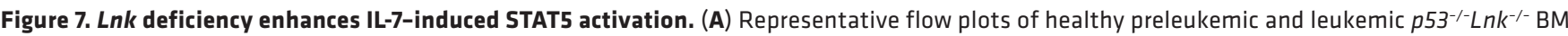
cells stained with of anti-B220 and intracellular IgM ( $\mu$-heavy chain, IC $\mu$ ) antibodies after fixation. (B) Combined B220 and intracellular IC $\mu$ staining divide $B$ cells into subpopulations at differentiation stages, which correlate with distinct IL-7 responsiveness. BM cells were starved, stimulated with or without IL-7, and subsequently fixed and stained as described in A, along with anti-pSTAT5 antibodies. Representative histograms of pSTAT5 signal for each population are shown. Dotted vertical lines indicate IL-7 control levels. Percentages of cells responded to IL-7 are indicated. $P$ values are calculated from 2-tailed Student's $t$ test when compared with WT cells stimulated by IL-7. (C and D) The dot plots show the percentages of pSTAT5

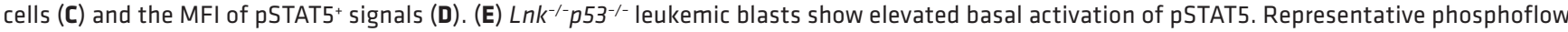
shows the activation status of basal-level pSTAT5 in BM B220 ${ }^{\circ} I C \mu^{-}$cells in comparison with leukemic cells after 1 hour of starvation. The dot plots show the quantification of relative MFI of phosphoflow signals. ${ }^{*} P<0.05$; ${ }^{* *} P<0.001$; ns, not significant, 2 -tailed Students' $t$ test. $n=4-10$ mice per group in all above experiments. (F) Lnk $\mathrm{K}^{-/} \mathrm{p} 53^{-/-}$leukemic blasts were starved and stimulated with or without IL-7 for 20 minuets. Cell lysates were subjected to WB analysis with indicated antibodies to phospho- or total JAKs. (G) HEK293T cells were transfected with mock or JAK3, along with either human or mouse Flag-tagged LNK expression constructs. Cell lysates were either precipitated with anti-Flag antibodies or directly and subjected to WB analysis with indicated antibodies. (H) BaF3/IL-7R cells were stably infected with retroviruses expressing either vector alone, or WT or R384E LNK mutant. Cell growth in the presence of IL-7 was quantified daily and graphed.

LNK negatively regulates normal and malignant HSC expansion and self-renewal in part by restricting the TPO/MPL/JAK2 signaling pathway (25-27). LNK has been reported to interact with Kit and downregulate SCF signaling in BM-derived mast cells (BMMCs) (23). LNK dysregulation has been found in MPNs and AMLs $(43,44)$, possibly due to its effects in MPL/JAK2 and KIT signaling pathways. A recent report showed that Lnk suppresses radiation resistance and radiation-induced $\mathrm{B}$ cell malignancies by inhibiting IL-11 signaling (45). IL-11 is critical for the ability of $\mathrm{Lnk}^{-/-}$HSPCs to recover from irradiation (IR) and become leukemic after a long latency. In contrast, our results demonstrate that LNK plays a direct role in $\mathrm{B}$ cell progenitors. It controls pro-B/ pre-B homeostasis and aging by regulating IL-7-mediated JAK/ STAT signaling. Furthermore, genetic studies suggest that the role of LNK in normal B cell and B-ALL development is independent of its effects in HSCs or early multipotential progenitors or CLPs, and it is independent of its role in the TPO/MPL/JAK2 pathway. This is further corroborated with our finding showing that pro-B cells from preleukemic mice are able to initiate B-ALL upon transplantation. Our studies thus implicate LNK as a regulator of IL-7R signaling in normal and malignant B progenitors.

Our data also suggest that $T p 53$ deficiency confers self-renewal ability in B progenitors, while Lnk deficiency confers cytokine hypersensitivity. The synergy between the loss of cytokine receptor signaling molecules and tumor suppressors is striking, and it is manifested by the fact that preleukemic B progenitors display enhanced self-renewal in vivo and in vitro and, further, enhanced basal and IL-7-stimulated STAT5 activation. Future investigation is warranted to elucidate the multiple underlying signaling pathways that function synergistically in ALLs.

To decipher mechanisms underlying high-risk ALL requires a multifaceted approach to provide insights into the earliest stages of cellular transformation. Identification of signaling events specific to distinct stages of malignant transformation and progression will help delineate a new understanding of B-ALL development and point to potentially druggable pathways for intervention. In this report, we characterized the B cell compartment of freshly isolated and unfractionated BM cells via a phosphoflow strategy that combines surface B cell marker and intracellular IgM staining with phospho-specific antibodies to separate subpopulations of B progenitor cells with distinct cytokine responsiveness. We found that Lnk deficiency expands pro-B cells that are the most responsive to IL-7, and synergistic loss of $L n k$ and Tp53 results in an enhanced STAT5 acti- vation in the preleukemic pro-B progenitors, indicating that LNK is a negative regulator for IL-7R-mediated JAK/SAT signaling that contributes to precursor B-ALL development. Fully transformed ALL blasts exhibited elevated activations of STAT5 at both basal conditions and upon stimulation. STAT5 is considered indispensable for maintenance of $B C R-A B L$-positive leukemia (46). Furthermore, overexpression of oncogenic STAT5 in the BM transplant models promotes B-ALL development in mice (47). Our signaling studies are translated into prolonged survival in Tp53-/ $L n k^{-/-}$B-ALL mice treated with JAK inhibition, supporting a previous report showing that the mice xenografted with human $L N K$ mutant B-ALLs are particularly sensitive to JAK inhibition (48).

Our work also uncovered an interaction between LNK and JAK3 that is activated by IL-7R. JAK3 and JAK1, but not JAK2, are activated in both normal $\mathrm{B}$ progenitors and $\mathrm{B}$ blasts. Thus, we believe that LNK interacts with JAK3 in B cell lineages, while it interacts with JAK2 in HSPCs. This finding is important, as LNK mutations co-occur with IL-7R mutations frequently (5 of 9), but not with CRLF2 rearrangement (1 of 9) or JAK2 mutations (0 of 9), in human Ph-like B-ALL (6). Although our results cannot exclude the possibility that LNK also plays a role in CRFL2r/ JAK2 mutant B-ALLs, our results point to distinct mechanisms for LNK in IL-7R-mediated signaling and proliferation in normal and malignant B progenitors. The co-occurrence of LNK and IL-7R mutations in human Ph-like ALLs also suggest JAK1/3 inhibitors would be effective in treating these patients to minimize the cytotoxic side effects of JAK2 inhibitors in suppressing hematopoiesis. Our biochemical studies found constitutive activation of multiple signaling pathways (e.g., STAT5, ERK, AKT, and S6), indicating that inhibition of these pathways simultaneously might be crucial. Indeed, we demonstrated that combined PI3K inhibition and JAK inhibition more potently suppressed B-ALL development in vivo and in vitro than single agents. Taken together, our studies enhance our understanding of the pathogenic mechanism underlying Ph-like B-ALLs with LNK mutations and will likely provide novel insights into therapeutic strategies.

\section{Methods}

Supplemental Methods are available online with this article.

Mice. $\mathrm{Mpl}^{-/-}$and $\mathrm{Lnk}^{-/-}$mice were provided by Frederic de Sauvage (49) (Genentech, South San Francisco, California, USA) and Tony Pawson (23) (Samuel Lunenfeld Research Institute, Toronto, Ontario, Canada), respectively. All mice were backcrossed onto the C57/BL6 

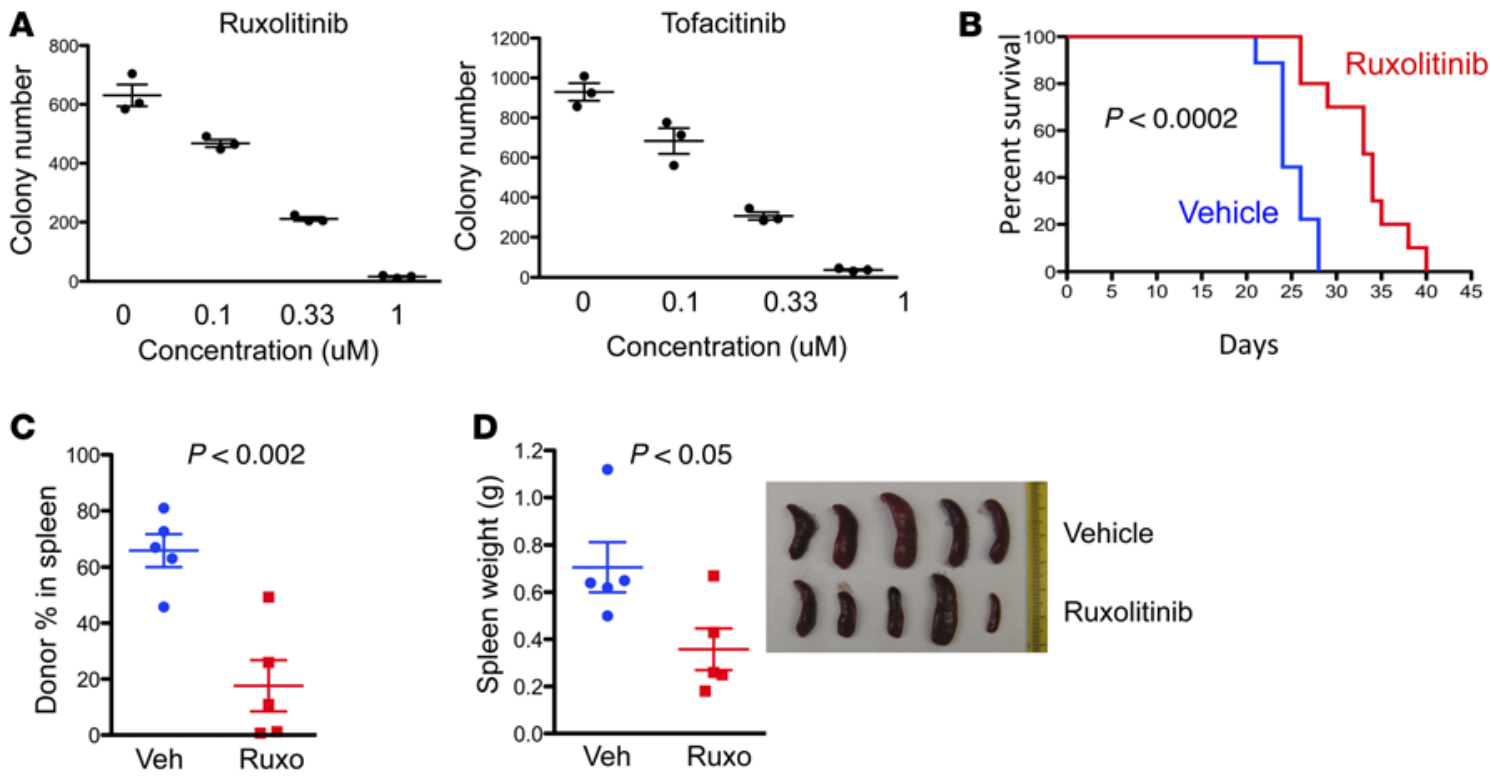

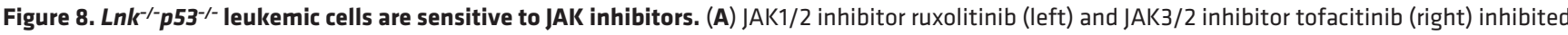
leukemic $L n k^{-1-} p 53^{-1-}$ B-ALL colony growth in a dose-dependent manner. Colony numbers are shown. (B) Cohort of mice transplanted with $L n k^{-1-} p 53^{-/-}$ B-ALLs were treated either with vehicle alone or ruxolitinib $(60 \mathrm{mg} / \mathrm{kg}$ ) daily 10 days after transplant. Kaplan-Meier survival curves are shown. $P$ value

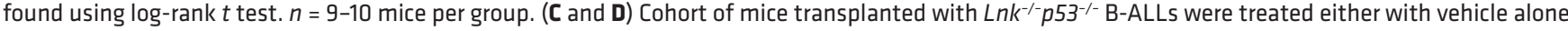
or ruxolitinib $(60 \mathrm{mg} / \mathrm{kg}$ ) daily for 10 days. The the donor leukemia percentage in the spleen (C) and spleen weight and images (D) are shown.

(CD45.2) background for over 9 generations. Tp53/-- mice on the C57/ BL6 background were purchased from the Jackson Laboratory. Both sexes were used in the studies. Young mice were 2-4 months old, and old mice were 12-16 months old.

Antibodies. Antibodies for cell surface staining are as follows: antiCD2 (clone RM2-5), anti-CD3e (clone 145-2C11), anti-CD5 (clone 53-7.3), anti-CD24 (clone M1/69), anti-CD25 (clone PC61.5), anti-CD43 (clone S7), anti-CD93 (clone AA4.1), anti-BP1 (clone 6C3), anti-B220 (clone RA3-6B2), anti-c-Kit (clone 2B8), anti-F4/80 (clone BM8), anti-Gr1 (clone RB6-8C5), anti-IgD (clone 11-26C), anti-IL-7R (clone A7R34), and anti-Mac1 (clone M1/70) were obtained from eBioscience; anti-CD4 (clone RM4-5), anti-CD8 $\alpha$ (clone 53-6.7), anti-CD19 (clone 1D3), anti-CD45.1 (clone A20), anti-CD45.2 (clone 104), anti-Flk2 (clone 2.4G2), anti-Ly6c (clone AL-21), anti-NK1.1 (clone PK136), antiSca1 (clone E13-161.7), and anti-Ter119 (clone TER-119) were obtained from BD Biosciences; and anti-CD150 (clone TC15-12F12.2) was obtained from BioLegend. FITC or APC-conjugate anti-IgM $\mu$-chain antibodies were purchased from Jackson ImmunoResearch Laboratories Inc. AlexaFluor 488-conjugate goat anti-rabbit IgG antibodies (catalog A-11034) were purchased from Molecular Probes.

Flow cytometry for cell surface markers, phosphoflow analysis, and cell sorting. For cell surface-flow cytometric analysis, cells were harvested from blood, BM, spleen, thymus, lymph nodes, and livers and stained with fluorochrome-conjugated antibodies to different cell markers for phenotypic analysis. For isolation of pro-B cells, BM samples were first depleted of lineage-positive cells using Biotin-conjugate anti-Ter119, anti-Mac-1, anti-Gr-1, anti-CD4, anti-CD5, and anti-CD8 $\alpha$ antibodies, followed by removal with Dynabeads (Invitrogen). The cells remaining after depletion were labeled with fluorochrome-conjugated anti-B220, anti-CD19, anti-CD43, anti-IgM $\mu$-chain, anti-NK1.1, anti-Ly6c, antiAA4.1, and/or anti-BP1 antibodies and sorted on FACS Aria. Pro-B cells
$\left(\mathrm{B}^{2} 2 \mathrm{O}^{+} \mathrm{CD} 19^{+} \mathrm{CD}^{2} 3^{+} \mathrm{AA} 4.1+\mathrm{IgM}^{-} \mathrm{NK} 11^{-} \mathrm{Ly}^{-} \mathrm{c}^{-}\right)$were used for BMT or culture, as well as for RNA isolation for microarray. For phosphoflow analysis, freshly isolated BM cells were starved for 2 hours in DMEM supplemented with $0.5 \%$ BSA and either left unstimulated or stimulated with murine IL-7 for 10 minutes. Cells were immediately fixed with $2 \%$ freshly prepared paraformaldehyde solution and permeabilized with ice-cold methanol. Cells were subsequently stained with anti-pSTAT5 (catalog 9351), anti-pAKT (catalog 4060), anti-pErk (catalog 4370), and anti-pS6 (catalog 4858) antibodies from Cell Signaling Technology, followed by staining with AlexaFluor 488-conjugate anti-rabbit IgG secondary antibodies, PE-conjugated anti-B220, and APC-conjugated anti-IgM $\mu$ chain and analysis on a BD FACSCanto II or Fortessa.

$I P$ and WB analysis. B-ALL cells from $T p 53^{--} L n k^{--}$mice were starved for 2 hours in DMEM medium (Invitrogen) plus 0.5\% BSA with either vehicle control or a graded dose of inhibitors for 0.5 hours, then stimulated with IL-7 and SCF (PeproTech) for 20 minutes. Cell lysates were subjected to standard WB analysis. Antibodies against pJAK1 (catalog 3331), JAK1 (catalog 3332), pJAK2 (catalog 3776), JAK2 (cata$\log 3230$ ), pJAK3 (catalog 5031), JAK3 (catalog 8863), pSTAT5 (catalog 9351), pErk (catalog 4370), Erk (catalog 9102), pAKT (catalog 4060), AKT (catalog 9272), pS6 (catalog 4858), S6 (catalog 2317), p4EBP1 (catalog 2855) and 4EBP1 (catalog 9644), pSTAT5 (catalog 4322), pAKT (catalog 4060), and pERK (catalog 4370) were from purchased Cell Signaling Technology. STAT5 (catalog sc-835) and $\beta$-actin (catalog sc-1616) antibodies were purchased from Santa Cruz Biotechnology.

293T cells were transfected with constructs expressing Flag-Lnk and JAK3. Two days after transfection, cells were lysed in buffer containing $0.5 \% \mathrm{NP}-40$, phosphatase, and protease inhibitors, as described previously (26). The protein supernatants were precipitated with antiFlag ( $5 \mu \mathrm{l}$ M2 beads, Sigma-Aldrich). The precipitates were blotted with anti-4G10 (0.5 $\mu \mathrm{g} / \mathrm{ml}$; catalog 05-321, EMD Millipore), JAK3 (1:1,000; 
A
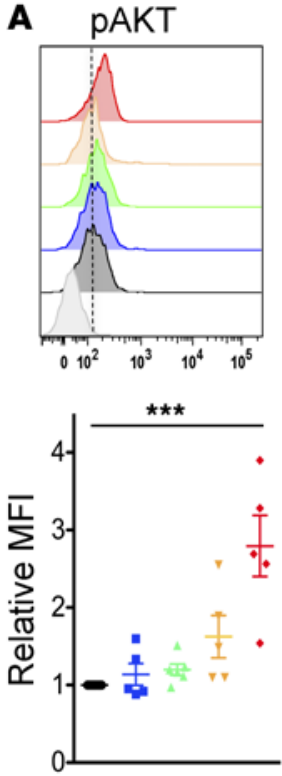

pS6
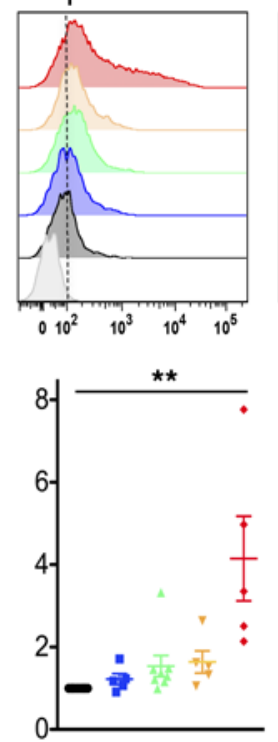

pERK
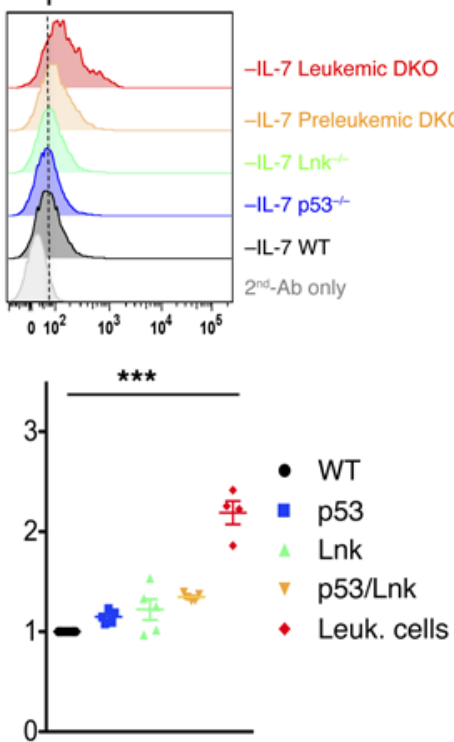

C

- - R B R+BR B R+BR BR+BR B R+B Drug $-++++++++++++\mathrm{IL}-7+\mathrm{SCF}$

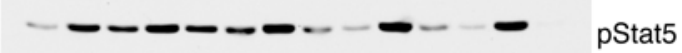

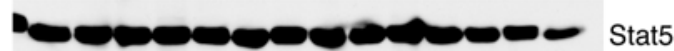

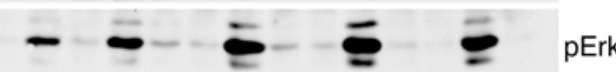

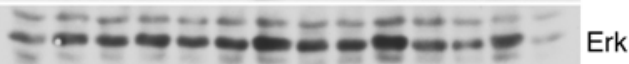

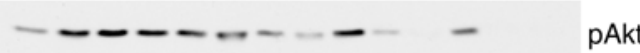

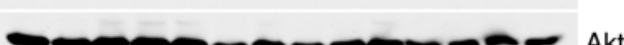

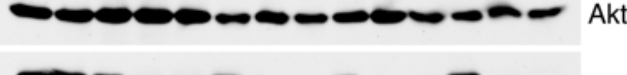

- -2 pS6

- - - - - - - - $\mathrm{s} 6$

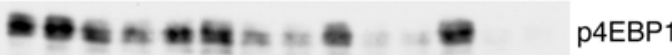

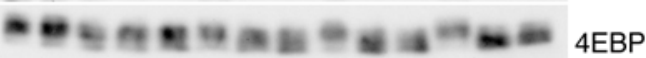

- - - - - - Actin
B

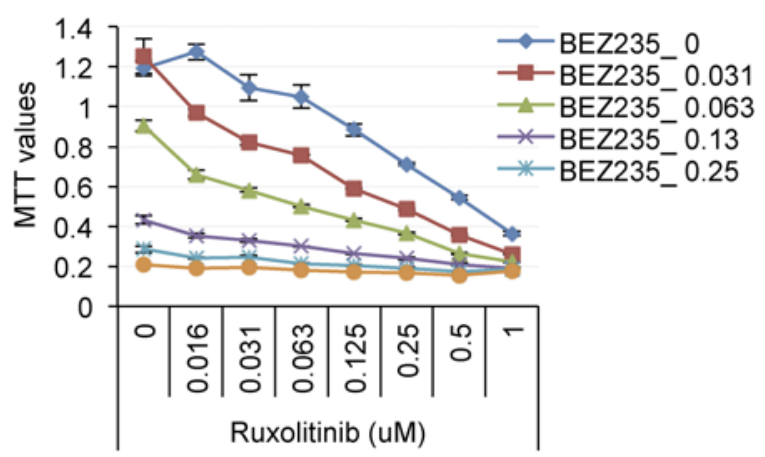

D

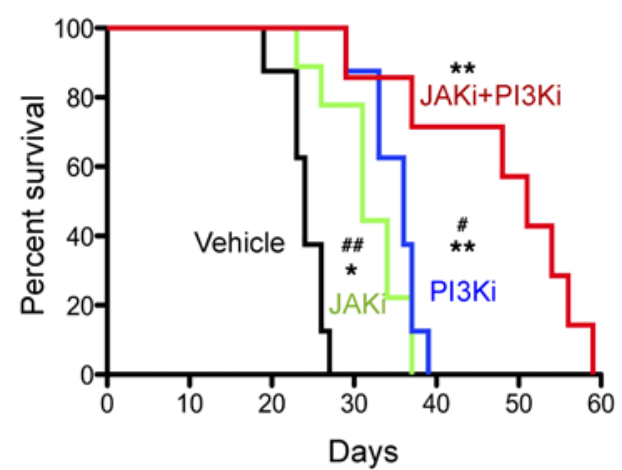

E

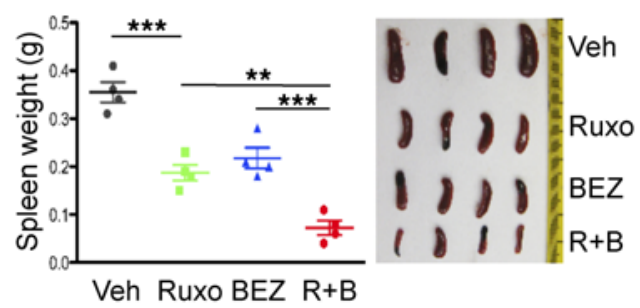

$\mathbf{F}$

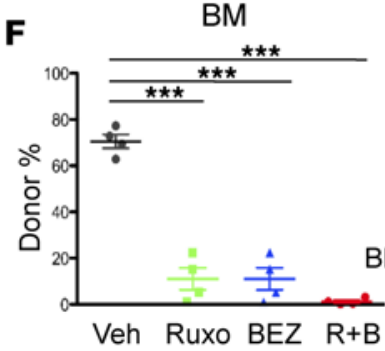

Spleen

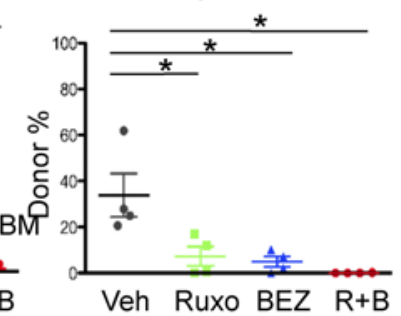

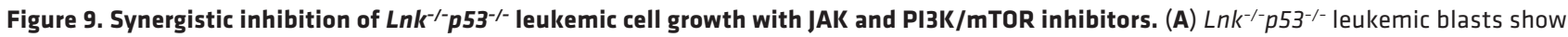
elevated basal activation of pAKT, pS6, and pERK. Representative phosphoflow shows the activation status of indicated proteins in BM B222 ${ }^{\circ}$ IC $\mu^{\text {med }}$ cells in comparison with leukemic cells after 1 hour of starvation. The dot plots show relative MFI of phosphoflow signals. $n=5-10 .{ }^{*} P<0.05 ;{ }^{*} P<$ 0.01 ; ${ }^{* *} P<0.001$, 2-tailed Student's $t$ test. $n=5-10$ mice per group. (B) Growth response curves for B-ALL cells treated with various concentrations of JAK and PI3K inhibitors. $L n K^{-1-p} 53^{-/-}$leukemic blasts were cultured in liquid culture supplemented with SCF and IL-7 in the presence of different concentrations of inhibitors, as indicated. Live cell numbers after 3 days of culture were determined by MTT absorbance. Representative results from 3 independent B-ALLs. (C) WB analyses for effector proteins of the STAT5, ERK, and PI3K/AKT pathways. Lnk ${ }^{-/-p} 53^{-/-}$leukemic blasts were stimulated with SCF and IL-7 for 20 minutes in the absence or presence of pretreatment with different concentrations of inhibitors. Cells were lysed, and the protein lysates were subjected to WB analysis with indicated antibodies. (D) Cohort of mice transplanted with $L n k^{-/-p} 53^{-/-}$B-ALLs were treated either with vehicle alone, ruxolitinib (JAKi), BEZ (PI3Ki), or combined 12 days after transplant. Kaplan-Meier survival curves are shown. Log-rank $t$ test, ${ }^{*} P<0.001$; ${ }^{*} P<0.0001$; drug treatment groups compared with vehicle group. ${ }^{*} P<0.01 ;{ }^{\# \#} P<0.005$, single drug treatment groups compared with combined drug treatment group. $n=7-9$ mice per group. (E and F) Cohort of mice transplanted with $L_{n k^{-/-}} p 53^{-/-}$B-ALLs were treated either with vehicle alone or ruxolitinib, BEZ, or combined daily for 10 days. The spleen weight along with the spleen images $(\mathbf{E})$, and the donor leukemia percentage in the BM (F, left) and the spleen (F, right) are shown. ${ }^{*} P<0.05 ;{ }^{* *} P<0.01$; ${ }^{* *} P<0.001$, 2-tailed Students' $t$ test. $n=4$ mice per group. 
catalog 8863, Cell Signaling Technology), or anti-Lnk (1:1,000; catalog AF5888, R\&D System) antibodies.

$B M$ transplantation (BMT). For leukemia transplant, sorted leukemic cells were injected retro-orbitally into sublethally irradiated (6 Gy, Orthovoltage Precision X-Ray) B6.SJL recipient mice. For transplantation of nonleukemic or preleukemic cells, sorted pro-B cells and LSK cells were mixed with $2 \times 10^{5}$ freshly isolated competitor cells and injected into lethally irradiated recipient mice (split dose of $10 \mathrm{~Gy}$ ). Two weeks to 6 months after transplantation, complete blood count (CBC) was measured using a Hemavet 950 (Drew Scientific), and hematocrits were measured on a hematocrit centrifuge (Micro-MB, IEC). PB, BM, and spleen cells from transplanted mice were analyzed for donor-derived fractions of myeloid, $\mathrm{T}$ cell, and B cell lineages with markers as indicated using flow cytometry.

Drug treatments in vitro and in vivo. For in vitro studies, ruxolitinib was purchased from LC Laboratories, while tofacitinib, BEZ235, selumetinib, trametinib, and other PI3K inhibitors were from Selleckchem. For in vivo studies, BEZ235 was purchased from LC Laboratories and ruxolitinib from Medkoo. All mice were injected with 3,000 leukemic blasts from $\mathrm{Lnk}^{-/} \mathrm{p} 53^{--}$B-ALLs BM with or without receiving $5.5 \mathrm{~Gy}$ sublethal IR. Similar results were obtained with or without IR. Ten or 12 days after injection, the mice were randomly divided into 2 (vehicle, ruxolitinib) or 4 (vehicle, ruxolitinib, BEZ235, and ruxolitinib/BEZ235) treatments with cohorts of 7-10 mice per group for survival curves. The mice were treated with vehicle only or $60 \mathrm{mg} / \mathrm{kg}$ body weight ruxolitinib dissolved in PEG300/5\% Glucose in water (v/v, 1:3) or $45 \mathrm{mg} / \mathrm{kg}$ BEZ235 in 10\% 1-methyl-2-pyrrolidone/90\% PEG 300 - or both ruxolitinib and BEZ235, by daily oral gavage - until the mice developed hind limb paralysis or became moribund. For spleen weight, donor percentage analysis, and signaling studies, cohorts of 4-5 mice per group were used. The mice were euthanized, and tissues were collected for further analysis 10 days after vehicle/drug treatments.

Colony assays. Colony assays were performed in semisolid methylcellulose (StemCell Technologies Inc.) according the manufacturer's recommendations. Total BM or spleen cells or sorted B cells were plated at appropriate concentrations in M3231 medium (StemCell Technologies Inc.), supplemented with murine IL-7 with or without SCF, and enumerated 7-8 days later. Serial replating assays were performed as previously described (50). In brief, primary colonies were recovered from the methylcellulose medium. Cells were washed with Iscove's modified Dulbecco's medium (IMDM, Invitrogen) with $2 \%$ FBS, and viable cells were enumerated with trypan blue. Under identical conditions, 30,000 cells $/ \mathrm{ml}$ were replated in each secondary plate. Colonies were scored after another 7-8 days.

Cellgrowth assay. Purified pro-B progenitor cells were plated at equal concentrations in a 96-well plate in DMEM medium supplemented with $10 \%$ FBS, supplemented with SCF and IL-7, over OP-9 stromal cells. Cells were counted, imunophenotyped by flow cytometry, and renewed every $2-5$ days. To measure leukemia cell proliferation, we seeded triplicate samples of cells $\left(100 \mu \mathrm{l} /\right.$ well; $\left.5 \times 10^{5} / \mathrm{ml}\right)$ in 96 -well plates and cultured them for 3 days with different concentrations of drugs. We added $15 \mu \mathrm{l}$ of 3-(4,5-dimethylthiazol-2-yl)-2, 5-diphenyl tetrazolium bromide (MTT; Promega) to each well and measured live cell numbers according to the manufacturer's instructions.

Histology and cytology. Femurs, tibias, spleens, thymus, lymph nodes, livers, and any abnormal tumor masses were fixed in $10 \%$ formalin (Sigma-Aldrich) and processed for paraffin blocks and sections.
H\&E stainings were used for histology. Blood smears and BM cytospins were fixed in cold methanol and stained with Wright-Giemsa (EMD Millipore) according to the manufacturer's recommendation. Cytology and histology images were taken using a Leica DM4000B microscope with plan FL2 $\times 10, \times 20$, or $\times 40$ objective lenses and a SPOT RT/SE Slider digital camera from SPOT Imaging Solutions.

Microarray. Purified pro-B cells from WT, Tp53-- $L n k^{-/-}$, and preleukemic $T p 53^{-/-} L n k^{-/-}$mice, along with leukemic B blasts from $T p 53^{-/-} L n k^{-/-}$ mice, were sorted directly into TRIzol LS (Invitrogen). RNA was isolated using microRNeasy kit (QIAGEN) and the microarray analysis was performed at the Penn Molecular Profiling/Genomics Facility using GeneChip Mouse Gene 2.0ST array (Affymetrix). Resulting expression data were normalized using robust multichip analysis (RMA) directly from the CEL files. Significant differential expression between the 2 groups was analyzed, and genes with significance analysis of microarrays (SAM) $P$ values less than 0.05 were selected. The microarray data can be accessed through the Gene Expression Omnibus (GEO GSE76940). Microarray data were tested for GSEA using MSigDb c2.cp v3.0 (37) (http://www.broad.mit.edu/gsea/). Briefly, Ph-like B-ALL gene signatures from the previous publication (6) were downloaded and added to the MSigDB C2 database, and GSEA analysis was subsequently performed using this database containing the Ph-like B-ALL signature.

Statistics. For all the experiments described, 2-tailed Student's $t$ tests were be performed with the data from 1 experiment with triplicates or from pooled data of 3-10 independent experiments. Graphs are presented as mean \pm SEM. Statistical analysis of Kaplan-Meier survival curves were preformed with log-rank tests using PRISM (Graphpad Software Inc.). $P$ values less than 0.05 were considered statistically significant.

Study approval. The animal studies were carried out in strict accordance with the recommendations in the Guide for the Care and Use of Laboratory Animals of the NIH. The protocol is approved by the Institutional Animal Care and Use Committee of the Children's Hospital of Philadelphia (72015-7-781).

\section{Author contributions}

YC designed and performed the experiments, analyzed the data, and wrote the manuscript. KC and JKC performed histology studies and analyzed pathology. CW and HZ performed the experiments and analyzed the data. DP performed peptide library screening. $\mathrm{AB}$ performed experiments and microarray analysis. WT conceived and designed the experiments, analyzed the data, and edited the manuscript.

\section{Acknowledgments}

W. Tong is supported by a NIH grant R01 HL095675, and D. Pei is supported by R01 GM062820. W. Tong received awards from the Gabrielle's Angel Foundation for Cancer Research and from Alex's Lemonade for Childhood Cancer Research and is a Leukemia Lymphoma Society (LLS) Scholar. Y. Cheng was supported by an American Heart Association (AHA) postdoctoral fellowship. H. Zhang was supported by T32HL007150. We would like to thank Shane Glasgow for his technical support of peptide library screening. We would like to thank the Human Hematopoietic Stem Cell Center of Excellence P30 DK090969 for its support of microarray analysis. We are grateful to Sarah Tasian, David Allman, Roger Greenberg, and Gerd Blobel for valuable discussions. 
Address correspondence to: Wei Tong, Abramson Bldg, Suite 316A, Children's Hospital of Philadelphia, 3615 Civic Center
Boulevard, Philadelphia, Pennsylvania 19104-4318, USA. Phone: 267.426.0930; E-mail: tongw@email.chop.edu.
1. Mullighan CG. Molecular genetics of B-precursor acute lymphoblastic leukemia. J Clin Invest. 2012;122(10):3407-3415.

2. Roberts KG, et al. Genetic alterations activating kinase and cytokine receptor signaling in highrisk acute lymphoblastic leukemia. Cancer cell. 2012;22(2):153-166

3. Harvey RC, et al. Identification of novel cluster groups in pediatric high-risk B-precursor acute lymphoblastic leukemia with gene expression profiling: correlation with genome-wide DNA copy number alterations, clinical characteristics, and outcome. Blood. 2010;116(23):4874-4884.

4. Loh ML, et al. Tyrosine kinome sequencing of pediatric acute lymphoblastic leukemia: a report from the Children's Oncology Group TARGET Project. Blood. 2013;121(3):485-488.

5. Schultz KR, et al. Risk- and response-based classification of childhood B-precursor acute lymphoblastic leukemia: a combined analysis of prognostic markers from the Pediatric Oncology Group (POG) and Children's Cancer Group (CCG). Blood. 2007;109(3):926-935.

6. Roberts KG, et al. Targetable kinase-activating lesions in Ph-like acute lymphoblastic leukemia. N Engl J Med. 2014;371(11):1005-1015.

7. Zhang J, et al. Key pathways are frequently mutated in high-risk childhood acute lymphoblastic leukemia: a report from the Children's Oncology Group. Blood. 2011;118(11):3080-3087.

8. Mullighan CG, et al. JAK mutations in high-risk childhood acute lymphoblastic leukemia. Proc Natl Acad Sci U S A. 2009;106(23):9414-9418.

9. Mullighan CG, et al. Rearrangement of CRLF2 in B-progenitor- and Down syndromeassociated acute lymphoblastic leukemia. Nat Genet. 2009;41(11):1243-1246.

10. Harvey RC, et al. Rearrangement of CRLF2 is associated with mutation of JAK kinases, alteration of IKZF1, Hispanic/Latino ethnicity, and a poor outcome in pediatric B-progenitor acute lymphoblastic leukemia. Blood. 2010;115(26):5312-5321.

11. Shochat C, et al. Gain-of-function mutations in interleukin-7 receptor-alpha (IL7R) in childhood acute lymphoblastic leukemias. J Exp Med. 2011;208(5):901-908.

12. Yoda A, et al. Functional screening identifies CRLF2 in precursor B-cell acute lymphoblastic leukemia. Proc Natl Acad Sci U S A. 2010;107(1):252-257.

13. Quentmeier H, et al. Cloning of human thymic stromal lymphopoietin (TSLP) and signaling mechanisms leading to proliferation. Leukemia. 2001;15(8):1286-1292.

14. Ziegler SF, Artis D. Sensing the outside world: TSLP regulates barrier immunity. Nat Immunol. 2010;11(4):289-293.

15. Levin SD, et al. Thymic stromal lymphopoietin: a cytokine that promotes the development of $\operatorname{IgM}^{+} \mathrm{B}$ cells and signals via a novel mechanism. JImmunol. 1999;162(2):677-683.

16. Isaksen DE, Baumann H, Trobridge PA, Farr AG, Levin SD, Ziegler SF. Requirement for stat5 in thymic stromal lymphopoietin-mediated signal transduction. J Immunol. 1999;163(11):5971-5977.

17. Isaksen DE, et al. Uncoupling of proliferation and Stat5 activation in thymic stromal lymphopoietin-mediated signal transduction. J Immunol. 2002;168(7):3288-3294.

18. Zhong J, et al. TSLP signaling network revealed by SILAC-based phosphoproteomics. Mol Cell Proteomics. 2012;11(6):M112 017764

19. Hertzberg L, et al. Down syndrome acute lymphoblastic leukemia, a highly heterogeneous disease in which aberrant expression of CRLF2 is associated with mutated JAK2: a report from the International BFM Study Group. Blood. 2010;115(5):1006-1017.

20. Bercovich D, et al. Mutations of JAK2 in acute lymphoblastic leukaemias associated with Down's syndrome. Lancet. 2008;372(9648):1484-1492.

21. Weigert O, et al. Genetic resistance to JAK2 enzymatic inhibitors is overcome by HSP9O inhibition. JExp Med. 2012;209(2):259-273.

22. Takaki S, et al. Control of B cell production by the adaptor protein lnk. Definition Of a conserved family of signal-modulating proteins. Immunity. 2000;13(5):599-609.

23. Velazquez L, et al. Cytokine signaling and hematopoietic homeostasis are disrupted in Lnk-deficient mice. J Exp Med. 2002;195(12):1599-1611.

24. Ema H, et al. Quantification of self-renewal capacity in single hematopoietic stem cells from normal and Lnk-deficient mice. Dev Cell. 2005;8(6):907-914.

25 . Seita J, et al. Lnk negatively regulates self-renewal of hematopoietic stem cells by modifying thrombopoietin-mediated signal transduction. Proc Natl Acad Sci U S A. 2007;104(7):2349-2354.

26. Bersenev A, Wu C, Balcerek J, Tong W. Lnk controls mouse hematopoietic stem cell self-renewa and quiescence through direct interactions with JAK2. J Clin Invest. 2008;118(8):2832-2844.

27. Buza-Vidas N, et al. Cytokines regulate postnatal hematopoietic stem cell expansion: opposing roles of thrombopoietin and LNK. Genes Dev. 2006;20(15):2018-2023.

28. Lin DC, et al. Adaptor protein Lnk binds to and inhibits normal and leukemic FLT3. Blood. 2012;120(16):3310-3317.

29. Zhang J, et al. The genetic basis of early T-cell precursor acute lymphoblastic leukaemia. Nature. 2012;481(7380):157-163.

30. Perez-Garcia A, et al. Genetic loss of SH2B3 in acute lymphoblastic leukemia. Blood. 2013;122(14):2425-2432.

31. Bersenev A, et al. Lnk constrains myeloproliferative diseases in mice. J Clin Invest. 2010;120(6):2058-2069.

32. Hof J, et al. Mutations and deletions of the TP53 gene predict nonresponse to treatment and poor outcome in first relapse of childhood acute lymphoblastic leukemia. J Clin Oncol. 2011;29(23):3185-3193.
33. Chiaretti S, et al. TP53 mutations are frequent in adult acute lymphoblastic leukemia cases negative for recurrent fusion genes and correlate with poor response to induction therapy. Haematologica. 2013;98(5):e59-e61.

34. Donehower LA, et al. Mice deficient for p53 are developmentally normal but susceptible to spontaneous tumours. Nature. 1992;356(6366):215-221.

35. Donehower LA, Lozano G. 20 years studying p53 functions in genetically engineered mice. Nat Rev Cancer. 2009;9(11):831-841.

36. Jacks $\mathrm{T}$, et al. Tumor spectrum analysis in p53-mutant mice. Curr Biol. 1994;4(1):1-7.

37. Subramanian A, et al. Gene set enrichment analysis: a knowledge-based approach for interpreting genome-wide expression profiles. Proc Natl Acad Sci US A. 2005;102(43):15545-15550.

38. Adolfsson J, et al. Identification of Flt $3^{+}$lymphomyeloid stem cells lacking erythro-megakaryocytic potential a revised road map for adult blood lineage commitment. Cell. 2005;121(2):295-306.

39. Kondo M, Weissman IL, Akashi K. Identification of clonogenic common lymphoid progenitors in mouse bone marrow. Cell. 1997;91(5):661-672.

40. Min H, Montecino-Rodriguez E, Dorshkind K. Effects of aging on the common lymphoid progenitor to pro-B cell transition. JImmunol. 2006;176(2):1007-1012.

41. Hardy RR, Carmack CE, Shinton SA, Kemp JD, Hayakawa K. Resolution and characterization of pro-B and pre-pro-B cell stages in normal mouse bone marrow. J Exp Med.1991;173(5):1213-1225.

42. Miller JP, Allman D. The decline in B lymphopoiesis in aged mice reflects loss of very early B-lineage precursors. JImmunol. 2003;171(5):2326-2330.

43. Oh ST, et al. Novel mutations in the inhibitory adaptor protein LNK drive JAK-STAT signaling in patients with myeloproliferative neoplasms. Blood. 2010;116(6):988-992.

44. Abdel-Wahab O. Genetics of the myeloproliferative neoplasms. Curr Opin Hematol. 2011;18(2):117-123.

45. Louria-Hayon I, et al. Lnk adaptor suppresses radiation resistance and radiation-induced B-cell malignancies by inhibiting IL-11 signaling. Proc Natl Acad Sci U S A . 2013;110(51):20599-20604.

46. Hoelbl A, et al. Stat5 is indispensable for the maintenance of bcr/abl-positive leukaemia. EMBO Mol Med.2010;2(3):98-110.

47. Joliot V, Cormier F, Medyouf H, Alcalde H, Ghysdael J. Constitutive STAT5 activation specifically cooperates with the loss of 53 function in B-cell lymphomagenesis. Oncogene. 2006;25(33):4573-4584.

48. Maude SL, et al. Targeting JAK1/2 and mTOR in murine xenograft models of Ph-like acute lymphoblastic leukemia. Blood. 2012;120(17):3510-3518.

49. Gurney AL, Carver-Moore K, de Sauvage FJ, Moore MW. Thrombocytopenia in c-mpl-deficient mice. Science. 1994;265(5177):1445-1447.

50. Smith KS, Rhee JW, Cleary ML. Transformation of bone marrow B-cell progenitors by E2a-Hlf requires coexpression of Bcl-2. Mol Cell Biol. 2002;22(21):7678-7687. 